Abstract The increasing level of experimental control over atomic and optical systems gained in the past years have paved the way for the exploration of new physical regimes in quantum optics and atomic physics, characterised by the appearance of quantum many-body phenomena, originally encountered only in condensed-matter physics, and the possibility of experimentally accessing them in a more controlled manner. In this review article we survey recent theoretical studies concerning the use of cavity quantum electrodynamics to create quantum manybody systems. Based on recent experimental progress in the fabrication of arrays of interacting micro-cavities and on their coupling to atomic-like structures in several different physical architectures, we review proposals on the realisation of paradigmatic many-body models in such systems, such as the BoseHubbard and the anisotropic Heisenberg models. Such arrays of coupled cavities offer interesting properties as simulators of quantum many-body physics, including the full addressability of individual sites and the accessibility of inhomogeneous mod-

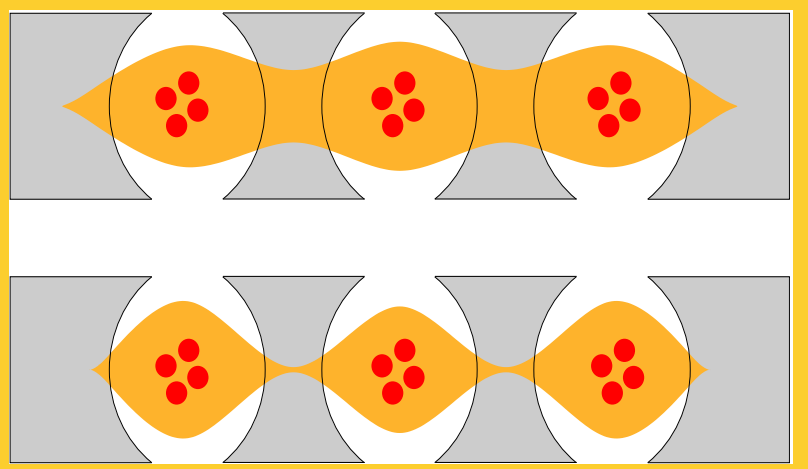

Crystal light: Photons (yellow) that ordinarily move freely through an array of cavities (top) might be frozen in place by their mutual repulsion (bottom). Such repulsion can be induced by laser pumping of suitable atoms (red) placed in the cavities. els.

\title{
Quantum Many-Body Phenomena in Coupled Cavity Arrays
}

\author{
Michael J. Hartmann ${ }^{1,2,3, *}$, Fernando G.S.L. Brandão $o^{2,3}$ and Martin B. Plenio ${ }^{2,3}$ \\ ${ }^{11}$ Technische Universität München, Physik Department I,James Franck Str., 85748 Garching, Germany \\ ${ }^{2}$ Institute for Mathematical Sciences, Imperial College London, SW7 2PG, United Kingdom \\ ${ }^{3}$ QOLS, The Blackett Laboratory, Imperial College London, Prince Consort Road, SW7 2BW, United Kingdom
}

'Received: ...

Published online: ...

Key words: quantum many-body models, polaritons, cavity QED, photon blockade

PACS: $42.50 . \mathrm{Dv}, 42.50 . \mathrm{Pq}, 03.67 . \mathrm{Bg}, 75.10 . \mathrm{Jm}$

\section{Contents}

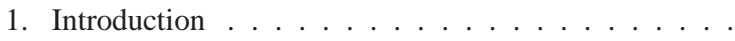

2. Cavity QED . . . . . . . . . . . . .

3. Coupled Cavity Arrays . . . . . . . . . . . . .

4. The Bose-Hubbard Model in Coupled Cavity Arrays .

5. Models Based on the Jaynes-Cummings Hamiltonian .

6. Phase Diagrams . . . . . . . . . . . . . . .

7. Effective Spin Models in Coupled Cavity Arrays . . .

8. Promising Platforms for Realisations . . . . . . . . 21 . . . . . . . . .

9. Applications . . . . . . . . . . . . . . . 24
10. Summary . . . . . . . . . . . . . . 24

\section{Introduction}

Quantum optics and atomic physics are concerned with the interaction of light and matter in the quantum regime, typically on the scale of a few atoms and light quanta. As atoms do not naturally interact strongly with each other and with light, the physics of such systems can usually be very well understood by ignoring collective effects and 
treating the interactions perturbatively. This is a much simpler and cleaner situation than the one encountered in the condensed matter context. There, strong interactions among the basic constituents, such as nuclei and electrons, lead to the emergence of completely new physics when one considers a mesoscopic or macroscopic number of interacting particles. Thus, even though the fundamental interactions between the constituent particles are usually known, it is challenging to fully describe the properties of such systems.

The higher level of isolation of quantum optical systems have permitted, over the past decades, the development of experimental techniques for their manipulation with an unprecedented level of control. Highlights include the invention of trapping and cooling techniques [1], which have found applications e.g. in spectroscopy and precision measurements and which culminated in the realisation of a Bose-Einstein condensate (BEC) of neutral atoms [2]; the manipulation of the state of individual atomic and photonic systems at the quantum level [3, 4] and its use in the study of the foundations of quantum theory [4,5]; and more recently the realisation of primitives for the processing of quantum information and for the implementation of quantum computation in several different quantum atomic and optical set-ups [6].

This progress raised the possibility of engineering strong interactions in these systems and of realising such strongly correlated models in large scales. This is of interest from a fundamental point of view, as in this way we can form new phases of matter for atoms, photons, or even molecules that do not exist outside the laboratory. Moreover, due to the high level of experimental control over them, it is possible to study many-body physics in a much cleaner manner than it is possible employing condensed-matter systems, in which the strong interaction with the environment and the very short space- and time-scales involved inhibit a precise control over their static and dynamic properties. The use of one quantum mechanical system to simulate the physics of a different one is an idea that dates back to Feynman [7] and has attracted considerable interest recently in the field of quantum information science [6].

A very successful example in this direction is the study of cold trapped atoms in optical lattices [8]. By loading a $\mathrm{BEC}$ of neutral atoms in a periodic optical lattice, formed by the off-resonant dipole interaction of atoms with overlapping standing waves of counter propagating lasers, the otherwise weak interactions of the atoms can be increased to such an extend that a strongly correlated many-body model is formed [9]. This idea led to several important experiments, such as the realisation of the Bose-Hubbard model [10] and of a Tonks-Girardeau gas [11] and is currently an active area of theoretical and experimental research [8, 12].

Also of note is the early study of strongly correlated many-body models in Josephson junctions arrays [13]. Although a Josephson junction is a mesoscopic solid state device, it behaves in many respects just like the type of systems studied in quantum optics, as the relevant physics can be understood by looking at a few quantum levels. In this context, arrays of interacting Josephson junctions have been used to reproduce properties of bosonic particles. For example, the Mott insulator phase [14] and the superconductor-to-Mott insulator transition [15] have been observed in Josephson junctions arrays.

Finally, theoretical proposals for using trapped ions for realising many-body models have been put forwarded [16 17, 18, 19 and a first benchmark experiment in this direction has been reported recently [20].

This article is devoted to review theoretical proposals for the use of the atom-light interaction in coupled microcavity arrays to create strongly correlated many-body models. This research was initiated in [21] and shortly after in [22,23] (see also [24]). Since then a substantial number of publications have extended these ideas in a variety of directions. These include photonic Mott insulators [25, 26], spin models [27, 28, 29, 30, 31, 32], dynamical effects [33, 34, 35, 36, 37], multi component models [38], phase diagrams [39, 40, 41, 42, 43], alternative physical realisations [44, 45, 46, 47, 48], experimental signatures [49,50] and quantum information applications [51, 52, 53, 54, 55, 56]. In fact, historically, some quantum information proposals [51] were presented before the theory for effective many-body models [21,22,23].

Recent experimental progress in the fabrication of microcavity arrays and the realisation of the quantum regime in the interaction of atomic-like structures and quantised electromagnetic modes inside those [57, 58, 59, 60, 61, 62, 63,64 opened up the possibility of using them as quantum simulators of many-body physics. The first motivation for this study is the desire to make photons or combined photonic-atomic excitations enter new quantum states, which do not naturally appear in nature. The second is that this set-up offer advantages over other proposals for the realisation of strongly interacting many-body models in quantum optical systems. Due to the small separations between neighbouring sites, it is experimentally very challenging to access individual sites in Josephson junctions arrays and optical lattices. Neighbouring sites in coupled cavity arrays, on the other hand, are usually separated by dozens of micrometres and can therefore be accurately accessed by optical frequencies. This allows e.g. the measurement of local properties as well as the exploration of inhomogeneous systems.

As the field of coupled cavity arrays is still quite young and many preprints are currently being reviewed, we have restricted ourselves here to only present details of work that has been published in peer reviewed journals before completion of the present text. This has been done in order to avoid any interferences with the policies of journals that consider the work in question for publication.

The organisation of the paper is as follows: In section 2 we discuss the use of cavities to enhance the interaction of light and matter and in section 3 we develop the basic description for an array of interacting cavities. The following section 4 reviews the theory for engineering effective Bose-Hubbard Hamiltonians with polaritons and 


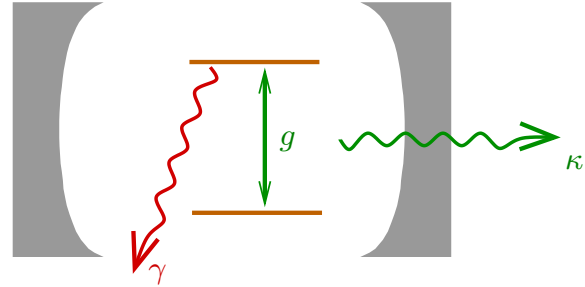

Figure 1 Cavity QED: A two level atom interacts via a dipole coupling $g$ with the photons in the cavity. Excitations a lost via spontaneous emission at a rate $\gamma$ and cavity decay at a rate $\kappa$.

photons. In section 5 we discuss a second class of effective Hamiltonians that exhibits a phase diagram similar to the Bose-Hubbard model: Jaynes-Cummings based many body models. The phase diagrams of both classes are then reviewed in section 6 Effective spin lattice Hamiltonians can also be generated in coupled cavity arrays and we review these approaches in section 7 In section 8 we then discuss several experimental platforms which appear to be suitable for a future implementation of the theory approach presented in this review. We finish with a summary in section 10

\section{Cavity QED}

Usually photons interact only over a very short time, and hence very weakly, with atoms. This interaction can be substantially increased if the atoms and photons are trapped inside a resonator. In the simplest situation, we can consider the coupling of a single light mode with a single two level system inside a cavity. Using the dipole and the rotating wave approximations their dynamics is governed by the Jaynes-Cummings Hamiltonian,

$$
H=\hbar \omega_{C} a^{\dagger} a+\hbar \omega_{0}|e\rangle\langle e|+\hbar g\left(a^{\dagger}|g\rangle\langle e|+a| e\rangle\langle g|\right),
$$

where $\omega_{C}, \omega_{0}$ are the frequencies of the resonant mode of the cavity and of the atomic transition, respectively, $g$ is Jaynes-Cummings coupling between the cavity mode and the two level system, $a^{\dagger}$ is the creation operator of a photon in the resonant cavity mode, and $|g\rangle,|e\rangle$ are the ground and excited states of the two level system. The Rabi frequency $g$ is given by

$$
g=\mathbf{d} f\left(r_{0}\right) \sqrt{\frac{\omega_{C}}{2 \epsilon_{0} V_{\text {mode }}}},
$$

where $V_{\text {mode }}$ is the mode volume of the cavity, $f\left(r_{0}\right)$ is the mode-function at the position $r_{0}$ of the two level system, $\mathbf{d}$ is the electric dipole transition moment, and $\epsilon_{0}$ is the free-space permittivity.

In addition to the coherent interaction, there are two main loss processes that affect the dynamics of the system: spontaneous emission from level $|e\rangle$ to level $|g\rangle$ at a rate $\gamma$ and leaking out of photons of the cavity mode at rate $\kappa$, see figure 1. The cavity decay rate $\kappa$ is connected to the quality factor of the cavity $Q$ by $Q=\omega_{C} /(2 \kappa)$. Although other processes, such as thermal motion of the atom or dephasing due to background electromagnetic fields, also contribute to the losses of the system, their effect are usually much smaller and can be disregarded in many applications.

The strong coupling regime of cavity QED is reached when the cooperativity factor, given by the the vacuum Rabi frequency to the square over the product of the spontaneous emission and cavity decay rates,

$$
\xi:=\frac{g^{2}}{2 \kappa \gamma},
$$

is much larger than one: $\xi \gg 1$. In this regime the coherent part of the evolution dominates over the decoherence processes and quantum dynamics of the joint atom cavity mode system can be observed. Such a regime has first been achieved in seminal experiments for microwave [4] and later optical frequencies [65], both using a single atom inside a Fabry-Pérot cavity formed between two miniature spherical mirrors. In these works, the strong coupling regime has been employed e.g. to create conditional quantum dynamics and entanglement between the photonic and atomic degrees of freedom [4,66] and to perform quantum non-demolition measurements [4]. Recently a strong coupling regime with very high cooperativity factors has also been achieved with artificial atoms formed by superconducting structures that interact with the electromagnetic modes of stripline resonator [62].

Most importantly for our purposes the strong coupling regime led to a realisation of the photon blockade effect [67], where, due to the strong interaction of the cavity mode with atoms, a single photon can modify the resonance frequency of the cavity mode in such a way that a second photon can not enter the cavity before the first leaks out.

In this review we are interested in the situation where several cavities operating in the strong coupling regime are coupled to each other. It turns out that the Fabry-Pérot architecture is not very suitable for the coupling of separate cavities. Nonetheless, several new cavity QED set-ups have recently emerged in which (i) the strong coupling regime has been achieved and (ii) the construction of arrays of coupled cavities has been realised, or at least seem reasonable to be so. These new systems, which we briefly review in section 8 are the strongest candidates for realising the proposals that we review in this paper.

\section{Coupled Cavity Arrays}

In this section we present the theoretical description for the coupling of cavities in an array. Photon hopping can occur between neighbouring cavities due to the overlap of the spacial profile of the cavity modes, see figure 2 . In 


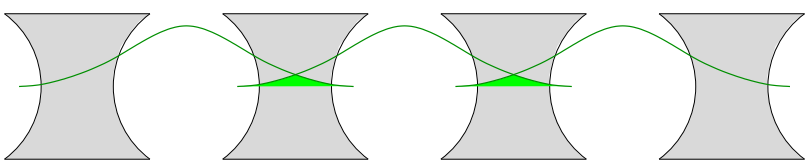

Figure 2 An array of coupled cavities. Photon hopping occurs due to the overlap (shaded green) of the light modes (green lines) of adjacent cavities.

order to model such a process, we follow references [21, 68, 69] and describe the array of cavities by a periodic dielectric constant, $\epsilon(\mathbf{r})=\epsilon(\mathbf{r}+R \mathbf{n})$, where $\mathbf{r}$ is a given three dimensional vector, $R$ is a constant and $\mathbf{n}$ labels tupels of integers. In the Coulomb gauge the electromagnetic field is represented by a vector potential $\mathbf{A}$ satisfying $\nabla \cdot(\epsilon(\mathbf{r}) \mathbf{A})=0$. We can expand $\mathbf{A}$ in Wannier functions, $\omega_{\mathbf{R}}$, each localised in a single cavity at location $\mathbf{R}=R \mathbf{n}$. We describe this single cavity by the dielectric function $\epsilon_{\mathbf{R}}(\mathbf{r})$. Then, from Maxwell's equations one can derive the eigenvalue equation

$$
\frac{\epsilon_{\mathbf{R}}(\mathbf{r}) \omega_{C}^{2}}{c^{2}} \mathbf{w}_{\mathbf{R}}-\nabla \times\left(\nabla \times \mathbf{w}_{\mathbf{R}}\right)=0,
$$

where the eigenvalue $\omega_{C}^{2}$ is the square the resonance frequency of the cavity, which is independent of $\mathbf{R}$ due to the periodicity. Assuming that the Wannier functions decay sufficiently fast outside the cavity, only Wannier modes of nearest neighbour cavities have a non-vanishing overlap.

Introducing the creation and annihilation operators of the Wannier modes, $a_{\mathbf{R}}^{\dagger}$ and $a_{\mathbf{R}}$, the Hamiltonian of the field can be written as

$$
\begin{aligned}
\mathcal{H} & =\omega_{C} \sum_{\mathbf{R}}\left(a_{\mathbf{R}}^{\dagger} a_{\mathbf{R}}+\frac{1}{2}\right) \\
& +2 \omega_{C} \alpha \sum_{<\mathbf{R}, \mathbf{R}^{\prime}>}\left(a_{\mathbf{R}^{\dagger}}^{\left.a_{\mathbf{R}^{\prime}}+\text { h.c. }\right) .}\right.
\end{aligned}
$$

Here $\sum_{<\mathbf{R}, \mathbf{R}^{\prime}>}$ is the sum of all pairs of cavities which are nearest neighbours. Since $\alpha \ll 1$, we neglected counter rotating terms which contain products of two creation or two annihilation operators of Wannier modes in deriving (5). The coupling parameter $\alpha$ is given by [69],

$$
\alpha=\int d^{3} r\left(\epsilon_{\mathbf{R}}(\mathbf{r})-\epsilon(\mathbf{r})\right) \mathbf{w}_{\mathbf{R}}^{\star} \mathbf{w}_{\mathbf{R}^{\prime}} ; \quad\left|\mathbf{R}-\mathbf{R}^{\prime}\right|=R,
$$

and has been obtained numerically for specific models [70].

In deriving Hamiltonian (5) we have assumed that all the cavities have the same resonant frequency and that the overlap of the Wannier modes $\alpha$ is the same for all cavitycavity interactions. In practise of course there will always be some disorder in the array and the resonance frequencies, $\omega_{C}(\mathbf{R})$, and tunnelling rates, $2 \omega_{C}(\mathbf{R}) \alpha((\mathbf{R})$ will differ from cavity to cavity. The disorder in the array can even give rise to interesting effects such as the emergence of glassy phases, see 6 .

\section{The Bose-Hubbard Model in Coupled Cavity Arrays}

Although realising Hamiltonian (5) might be interesting for quantum information propagation [71,72], it is not the type of model that one would like to quantum simulate. Indeed, the model is harmonic and can be very easily solved in terms of Bloch waves, which allows for a simple understanding of all its basic properties. The situation changes dramatically if we add an on-site interaction term. The interplay of tunnelling and interaction leads to interesting many-body physics. A paradigmatic model for such a situation is the Bose-Hubbard Hamiltonian, which reads

$$
\begin{array}{r}
H_{B H}=\mu \sum_{k} b_{k}^{\dagger} b_{k}-J \sum_{<k, k^{\prime}>}\left(b_{k}^{\dagger} b_{k^{\prime}}+\text { h.c. }\right) \\
+U \sum_{k} b_{k}^{\dagger} b_{k}\left(b_{k}^{\dagger} b_{k}-1\right),
\end{array}
$$

where $b_{k}^{\dagger}$ creates a boson at site $k, J$ is the hopping rate, $U$ the on-site interaction strength, and $\mu$ the chemical potential. This model, which was first discussed by Fisher et al in Ref. [73,74] as the bosonic counterpart of the Hubbard model [75], contains two different phases at zero temperature that are separated by a quantum phase transition where the ration $U / J$ crosses a critical value. Let us briefly describe the two phases qualitatively.

When $J \gg U$, the tunnelling term dominates and the lowest energy state of the system is a condensate of delocalised bosons: the system is in a superfluid phase. If we start to increase a repulsive on-site interaction, adding more than one particle to one site requires energy that grows quadratically with the particle number. It will thus be energetically favourable for the bosons to evenly distribute across all lattice sites and avoid moving around in order to minimise the repulsion. Above a specific value of $U / J$, the system ceases to be a superfluid and becomes a Mott insulator, with a well defined number of localised particles in each site 1 . The superfluid-to-Mott insulator phase transition is of a quantum character and is driven by quantum fluctuations, as it occurs even at zero temperature. An order parameter for such a transition is the variance of the number of bosons in a given site, $\left\langle\left(b_{k}^{\dagger} b_{k}-\left\langle b_{k}^{\dagger} b_{k}\right\rangle\right)^{2}\right\rangle$. In the superfluid phase, the number of bosons in each site fluctuates and thus the variance has a non-zero value. In the Mott insulator phase, in turn, the variance is close to zero, as the bosons tend to be localised in each site.

As we saw in section 3 the coupling between neighbouring cavities naturally leads to the tunnelling term for photons in arrays of coupled cavities. Hence, for a realisation of a Bose-Hubbard model a way to effectively generate an interaction term is needed. Indeed, up to harmonic terms which can easily be compensated for, the Bose-Hubbard interaction term has the form of a Kerr self non-linearity

\footnotetext{
${ }^{1}$ In the case of incommensurate filling factors, i.e. if the number of particles is not a multiple of the number of lattice sites, the system will always be in a superfluid phase.
} 


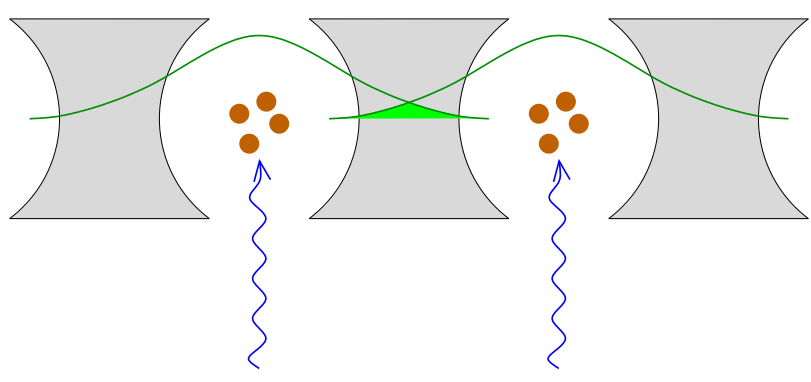

Figure 3 An array of cavities as described by our model. Photon hopping occurs due to the overlap (shaded green) of the light modes (green lines) of adjacent cavities. Atoms in each cavity (brown), which are driven by external lasers (blue) give rise to an on site potential. Reprinted with permission from [21].

$\left(b_{k}^{\dagger}\right)^{2} b_{k}^{2}$. Although such an interaction naturally appears in some media as a result of a non-zero third order electric susceptibility, its effect is negligible on the level of individual quanta, which is a reason for the difficulty of realising nonlinear optics for individual quantum systems. Using the enhanced light-matter interaction in cavity QED, it is possible to engineer much stronger nonlinearities. Intuitively the strong interactions of the light mode with atoms inside the cavity, under particular circumstances, mediates strong nonlinear interaction among the photons of the cavity mode. The strength of the nonlinearity can be increased even further if instead of considering photons as the bosonic particles of the model, one considers polaritons, joint photonicatomic excitations. In the next section we review the proposal for realising the Bose-Hubbard Hamiltonian using such polaritons.

\subsection{Polaritonic Bose-Hubbard model}

The polaritonic Bose-Hubbard model is hosted in an array of cavities that are filled with atoms of a particular 4 level structure, which are driven with an external laser, see figures 3 and 4 . Thereby the laser drives the atoms in the same manner as in Electromagnetically Induced Transparency (EIT) [76]: The transitions between levels 2 and 3 are coupled to the laser field and the transitions between levels 2-4 and 1-3 couple via dipole moments to the cavity resonance mode. Levels 1 and 2 are assumed to be metastable and their spontaneous emission rates are thus negligible.

It has been shown by Imamŏglu and co-workers, that this atom cavity system can exhibit a very large nonlinearity [77, 78, 79, 80], and a similar nonlinearity has been observed experimentally [67].

Considering the level structure of figure 4 in a rotating frame with respect to $H_{0}=\omega_{C}\left(a^{\dagger} a+\frac{1}{2}\right)+$ $\sum_{j=1}^{N}\left(\omega_{C} \sigma_{22}^{j}+\omega_{C} \sigma_{33}^{j}+2 \omega_{C} \sigma_{44}^{j}\right)$, the Hamiltonian of

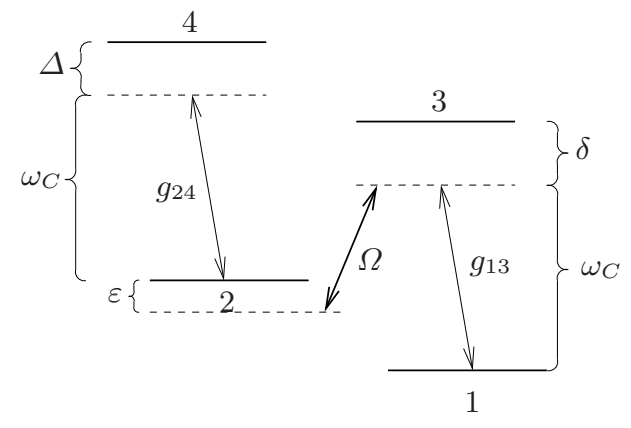

Figure 4 The level structure and the possible transitions of one atom, $\omega_{C}$ is the frequency of the cavity mode, $\Omega$ is the Rabi frequency of the driving by the laser, $g_{13}$ and $g_{24}$ are the parameters of the respective dipole couplings and $\delta, \Delta$ and $\varepsilon$ are detunings. Reprinted with permission from [21].

the atoms in the cavity reads,

$$
\begin{aligned}
H^{I} & =\sum_{j=1}^{N}\left(\varepsilon \sigma_{22}^{j}+\delta \sigma_{33}^{j}+(\Delta+\varepsilon) \sigma_{44}^{j}\right) \\
& +\sum_{j=1}^{N}\left(\Omega \sigma_{23}^{j}+g_{13} \sigma_{13}^{j} a^{\dagger}+g_{24} \sigma_{24}^{j} a^{\dagger}+\text { h.c. }\right)
\end{aligned}
$$

where $\sigma_{k l}^{j}=\left|k_{j}\right\rangle\left\langle l_{j}\right|$ projects level $l$ of atom $j$ to level $k$ of the same atom, $\omega_{C}$ is the frequency of the cavity mode, $\Omega$ is the Rabi frequency of the driving by the laser and $g_{13}$ and $g_{24}$ are the parameters of the dipole coupling of the cavity mode to the respective atomic transitions.

In a cavity array, $N$ atoms in each cavity couple to the cavity mode via the interaction $H^{I}$ and photons tunnel between neighbouring cavities as described in equation (5). Hence the full Hamiltonian describing this system reads

$$
\begin{aligned}
H & =\sum_{\mathbf{R}} H_{\mathbf{R}}^{I}+\omega_{C} \sum_{\mathbf{R}}\left(a_{\mathbf{R}}^{\dagger} a_{\mathbf{R}}+\frac{1}{2}\right) \\
& +2 \omega_{C} \alpha \sum_{<\mathbf{R}, \mathbf{R}^{\prime}>}\left(a_{\mathbf{R}^{\dagger}}^{\left.a_{\mathbf{R}^{\prime}}+\text { h.c. }\right)}\right.
\end{aligned}
$$

and can emulate a Bose-Hubbard model for polaritons as we will see in the following.

Assuming that all atoms interact in the same way with the cavity mode, the description can be restricted to Dicke type dressed states, in which the atomic excitations are delocalised among all the atoms. In the case where $g_{24}=$ 0 and $\varepsilon=0$, level 4 of the atoms decouples from the dressed-state excitation manifolds [79]. The Hamiltonian (8) can then be expressed in terms of the following creation (and annihilation) operators:

$$
\begin{aligned}
& p_{0}^{\dagger}=\frac{1}{B}\left(g S_{12}^{\dagger}-\Omega a^{\dagger}\right), \\
& p_{ \pm}^{\dagger}=\sqrt{\frac{2}{A(A \pm \delta)}}\left(\Omega S_{12}^{\dagger}+g a^{\dagger} \pm \frac{A \pm \delta}{2} S_{13}^{\dagger}\right),
\end{aligned}
$$


where $g=\sqrt{N} g_{13}, B=\sqrt{g^{2}+\Omega^{2}}, A=\sqrt{4 B^{2}+\delta^{2}}$, $S_{12}^{\dagger}=\frac{1}{\sqrt{N}} \sum_{j=1}^{N} \sigma_{21}^{j}$ and $S_{13}^{\dagger}=\frac{1}{\sqrt{N}} \sum_{j=1}^{N} \sigma_{31}^{j}$.

The operators $p_{0}^{\dagger}, p_{+}^{\dagger}$ and $p_{-}^{\dagger}$ describe polaritons, quasi particles formed by combinations of atom and photon excitations. In the subspace spanned by symmetric Dicke states and in the limit of large atom numbers, $N \gg 1$, they satisfy bosonic commutation relations,

$$
\left[p_{j}, p_{l}\right]=0 \text { and }\left[p_{j}, p_{l}^{\dagger}\right]=\delta_{j l} \text { for } j, l=0,+,-.
$$

$p_{0}^{\dagger}, p_{+}^{\dagger}$ and $p_{-}^{\dagger}$ thus describe independent bosonic particles. In terms of these polaritons, the Hamiltonian (8) for $g_{24}=$ 0 and $\varepsilon=0$ reads,

$$
\left[H^{I}\right]_{g_{24}=0, \varepsilon=0}=\mu_{0} p_{0}^{\dagger} p_{0}+\mu_{+} p_{+}^{\dagger} p_{+}+\mu_{-} p_{-}^{\dagger} p_{-},
$$

where the frequencies are given by $\mu_{0}=0, \mu_{+}=(\delta-$ A) $/ 2$ and $\mu_{-}=(\delta+A) / 2$.

The polaritons $p_{0}$ only contain atomic contributions in the two metastable states 1 and 2 but not in level 3 , which shows spontaneous emission. Hence, these polaritons do not populate radiating atomic levels and are thus called dark state polaritons [76].

As we will discuss now, the dynamics of the dark state polaritons $p_{0}$ decouples from the dynamics of the remaining species $p_{+}$and $p_{-}$for a suitable parameter regime. In this regime the frequency $\mu_{0}$ is sufficiently separated from $\mu_{+}$and $\mu_{-}$, such that neither the interaction with the atomic level 4 nor the photon tunnelling between cavities can induce any mixing between the polariton species $p_{0}$ and $p_{ \pm}$.

\subsubsection{Polariton-polariton interactions and polariton tunnelling}

The polaritons $p_{0}^{\dagger}$ interact with each other via a self Kerr nonlinearity. To derive this interaction, one first needs to write the full Hamiltonian $H^{I}$, (8), in the polariton basis, expressing the operators $\sum_{j=1}^{N} \sigma_{22}^{j}$ and $a^{\dagger} \sum_{j=1}^{N} \sigma_{24}^{j}$ in terms of $p_{0}^{\dagger}, p_{+}^{\dagger}$ and $p_{-}^{\dagger}$. The coupling of the polaritons to the level 4 of the atoms via the dipole moment $g_{24}$ reads,

$$
g_{24}\left(\sum_{j=1}^{N} \sigma_{42}^{j} a+\text { h.c. }\right) \approx-g_{24} \frac{g \Omega}{B^{2}}\left(S_{14}^{\dagger} p_{0}^{2}+\text { h.c. }\right),
$$

where $S_{14}^{\dagger}=\frac{1}{\sqrt{N}} \sum_{j=1}^{N} \sigma_{41}^{j}$. In deriving [13), a rotating wave approximation was used: In a frame rotating with respect to 12 , the polaritonic creation operators rotate with the frequencies $\mu_{0}, \mu_{+}$and $\mu_{-}$. Furthermore, the operator $S_{14}^{\dagger}$ rotates at the frequency $2 \mu_{0}$, (c.f. $[8$ ). Hence, provided that

$$
\left|g_{24}\right|,|\varepsilon|,|\Delta| \ll\left|\mu_{+}\right|,\left|\mu_{-}\right|
$$

all terms that rotate at frequencies $2 \mu_{0}-\left(\mu_{+} \pm \mu_{-}\right)$or $\mu_{0} \pm \mu_{+}$or $\mu_{0} \pm \mu_{-}$can be neglected, which eliminates all interactions that would couple $p_{0}^{\dagger}$ and $S_{14}^{\dagger}$ to the remaining polariton species.

For $\left|g_{24} g \Omega / B^{2}\right| \ll|\Delta|$, the coupling to level 4 can be treated perturbatively. This results in an energy shift of $2 U$ with

$$
U=-\frac{g_{24}^{2}}{\Delta} \frac{N g_{13}^{2} \Omega^{2}}{\left(N g_{13}^{2}+\Omega^{2}\right)^{2}}
$$

and in an occupation probability of the state of one $S_{14}^{\dagger}$ excitation of $-2 U / \Delta$, which determines an effective decay rate for the polariton $p_{0}^{\dagger}$ via spontaneous emission from level 4. Note that $U>0$ for $\Delta<0$ and vice versa. In a similar way, the two photon detuning $\varepsilon$ leads to an energy shift of $\varepsilon g^{2} B^{-2}$ for the polariton $p_{0}^{\dagger}$, which plays the role of a chemical potential in the effective Hamiltonian.

Hence, provided (14) holds, the Hamiltonian for the dark state polariton $p_{0}^{\dagger}$ can be written as

$$
H_{\mathrm{eff}}=U\left(p_{0}^{\dagger}\right)^{2}\left(p_{0}\right)^{2}+\varepsilon \frac{g^{2}}{B^{2}} p_{0}^{\dagger} p_{0},
$$

in the rotating frame.

Let us now look at an array of interacting cavities, each under the conditions discussed above. The first term of the Hamiltonian (5) has already been incorporated in the polariton analysis for one individual cavity. The second term, which describes photon tunnelling, transforms into the polariton picture via (10). To distinguish between the dark state polaritons in different cavities, we introduce the notation $p_{\mathbf{R}}^{\dagger}$ to label the polariton $p_{0}^{\dagger}$ in the cavity at position $\mathbf{R}$. The photon hopping translates to polariton hoppings according to,

$$
\begin{aligned}
a_{\mathbf{R}}^{\dagger} a_{\mathbf{R}^{\prime}} & \approx \frac{\Omega^{2}}{B^{2}} p_{\mathbf{R}}^{\dagger} p_{\mathbf{R}^{\prime}} \\
& + \text { "terms for other polariton species". }
\end{aligned}
$$

Contributions of different polaritons decouple due to the separation of their frequencies $\mu_{0}, \mu_{+}$and $\mu_{-}$. As a consequence the Hamiltonian for the polaritons $p_{\mathbf{R}}^{\dagger}$ takes on the form (7), with

$$
J=\frac{2 \omega_{C} \Omega^{2}}{N g_{13}^{2}+\Omega^{2}} \alpha,
$$

$U$ as given by equation (15) and $\mu=\epsilon g^{2} / B^{2}$.

4.1.2. Polariton lifetime: spontaneous emission and cavity decay

We now discuss the loss sources in the system. Levels 1 and 2 are metastable and therefore have negligible decay rates on the relevant time scales. The decay mechanism for the dark state polariton then originates mainly from photons that leak out of the cavity and from the small, but nonzero, population of level 4 , which leads to spontaneous 
emission. The resulting effective decay rate for the dark state polariton reads

$$
\Gamma_{0}=\frac{\Omega^{2}}{B^{2}} \kappa+\Theta(n-2) \frac{g_{24}^{2} g^{2} \Omega^{2}}{\Delta^{2} B^{4}} \gamma_{4},
$$

where $\kappa$ is the cavity decay rate, $\gamma_{4}$ the spontaneous emission rate from level $4, n$ is the average number of photons, and $\Theta$ the Heaviside step function. Assuming that $g_{13}=g_{24}$, the maximal achievable rate $U / \Gamma_{0}$ can be readily seen to be $g_{13} / \sqrt{4 \kappa \Theta(n-2) \gamma_{4}}$. Hence, for large cooperativity factors, $\xi \gg 1$, ratios of $U / \Gamma_{0} \gg 1$ can be achieved, which ensures that the dynamics of the BoseHubbard model can be observed under realistic conditions.

\subsubsection{The phase transition}

Of great interest is of course whether the quantum phase transition from a Mott insulator to a superfluid state could be observed with the present polariton approach. In a system with on average one polariton per cavity, the Mott insulator state is characterised by the fact that the local state in each cavity is a single polariton Fock state with vanishing fluctuations of the polariton number. The superfluid phase in contrast shows polariton number fluctuations.

Figure 5] shows numerical simulations of the full dynamics of an array of three cavities as described by equation (9), including spontaneous emission and cavity decay, and studies the number of polaritons in one cavity/site, $n_{l}=\left\langle p_{l}^{\dagger} p_{l}\right\rangle$ for site $l$, and the number fluctuations, $F_{l}=$ $\left\langle\left(p_{l}^{\dagger} p_{l}\right)^{2}\right\rangle-\left\langle p_{l}^{\dagger} p_{l}\right\rangle^{2}$. A comparison between the full model (9) and the effective model (7) is done by considering the differences in the occupation numbers, $\delta n_{l}=\left[n_{l}\right]_{\text {cavities }}-$ $\left[n_{l}\right]_{\mathrm{BH}}$, and number fluctuations, $\delta F_{l}=\left[F_{l}\right]_{\text {cavities }}-\left[F_{l}\right]_{\mathrm{BH}}$, at each time step [21]. Initially there is exactly one polariton in each cavity and parameters for toroidal microcavities from [81], $g_{24}=g_{13}=2.5 \times 10^{9} s-1, \gamma_{4}=\gamma_{3}=$ $1.6 \times 10^{7} s^{-1}$ and $\kappa=0.4 \times 10^{5} s^{-1}$, are assumed. As the system is driven from a Mott insulator state to a superfluid state by ramping up the driving laser $\Omega$, the particle number fluctuations increase significantly. The numerics in figure 5 show good agreement of the full dynamics as described by equation (9) and the dynamics of the corresponding Bose-Hubbard model (7) and thus confirm the possibility of observing the Mott insulator-to-superfluid transition in such a system. The oscillatory behaviour is related to the fact that the initial state of the system is, due to the nonzero tunnelling rate $J$, not its ground state. This initial state has been chosen since it's preparation in an experiment is expected to be easier compared to other states and figure 5 thus shows the dynamics that is expected to be observed in an experiment.

The phase diagram for this model and its dependence on the atom number $N$ have been analysed in [39], where also a glassy phase has been predicted for a system with disorder, see section 6 for details.
A polariton model which generates an effective LiebLiniger Hamiltonian and is capable of reproducing the Tonks Girardeau regime was proposed in [45]. The scheme employs atoms of the same level structure as in figure 4 that couple to light modes of a tapered optical fibre. Furthermore quantum phase transitions of Dicke-model in cavity QED have been analysed [82].

\subsubsection{Two component Bose-Hubbard model}

Before we conclude this section, let us briefly review the proposal of Ref. [38] for creating a two component polaritonic Bose-Hubbard model. This model, which describes the dynamics of two independent bosonic particles in each site, reads

$$
\begin{aligned}
H_{\mathrm{eff}} & =\sum_{k, j=b, c} \mu_{j} n_{k}^{(j)}-\sum_{<k, k^{\prime}>; j, l=b, c} J_{j, l}\left(j_{k}^{\dagger} l_{k^{\prime}}+\text { h.c. }\right) \\
& +\sum_{k, j=b, c} U_{j} n_{k}^{(j)}\left(n_{k}^{(j)}-1\right)+\sum_{k} U_{b, c} n_{k}^{(b)} n_{k}^{(c)},(20)
\end{aligned}
$$

where $b_{k}^{\dagger}\left(c_{k}^{\dagger}\right)$ create polaritons of the type $b(c)$ in the cavity at site $k, n_{k}^{(b)}=b_{k}^{\dagger} b_{k}$ and $n_{k}^{(c)}=c_{k}^{\dagger} c_{k} \cdot \mu_{b}$ and $\mu_{c}$ are the polariton energies, $U_{b}, U_{c}$ and $U_{b, c}$ their on-site interactions and $J_{b, b}, J_{c, c}$ and $J_{b, c}$ their tunnelling rates. This model exhibits interesting quantum many-body phenomena such as spin density separation [83], spin order in the Mott regime [84] and phase separation [85].

The Hamiltonian (20) can be realised in the same setup as above, by considering the atom-photon interactions in a dispersive regime with $\delta \gg \Omega, g$. Then, the polariton operators 10 read,

$$
\begin{array}{ll}
p_{0}^{\dagger}=\frac{1}{B}\left(g S_{12}^{\dagger}-\Omega a^{\dagger}\right) & \mu_{0}=0 \\
p_{-}^{\dagger} \approx \frac{1}{B}\left(\Omega S_{12}^{\dagger}+g a^{\dagger}\right)-\frac{B}{\delta} S_{13}^{\dagger} & \mu_{-}=-\frac{B^{2}}{\delta} \\
p_{+}^{\dagger} \approx S_{13}^{\dagger}+\frac{1}{\delta}\left(\Omega S_{12}^{\dagger}+g a^{\dagger}\right) & \mu_{+}=\delta+\frac{B^{2}}{\delta}
\end{array}
$$

to leading order in $\delta^{-1}$. Note that now, not only the polariton $p_{0}$, but also $p_{-}^{\dagger}$ do not experience loss from spontaneous emission to leading order in $\delta^{-1}$. One can therefore define two dark state polaritons species

$$
b^{\dagger}=\frac{1}{B}\left(g S_{12}^{\dagger}-\Omega a^{\dagger}\right) ; \quad c^{\dagger}=\frac{1}{B}\left(\Omega S_{12}^{\dagger}+g a^{\dagger}\right) .
$$

From an analysis similar to the one outlined above for the one component case, it can be shown that the dynamics of these two polaritons species is given by the two component Bose-Hubbard model (20), where the parameters are given by, $J_{b b}=\alpha \frac{g^{2}}{B^{2}}, J_{c c}=\alpha \frac{\Omega^{2}}{B^{2}}, J_{b c}=\alpha \frac{g \Omega}{B^{2}}, U_{b}=-\frac{g_{24}^{2} g^{2} \Omega^{2}}{B^{4} \Delta}$, $U_{c}=-\frac{g_{24}^{2} g^{2} \Omega^{2}}{B^{4}\left(\Delta+2 B^{2} / \delta\right)}$ and $U_{b c}=-\frac{g_{24}^{2}\left(g^{2}-\Omega^{2}\right)^{2}}{B^{4}\left(\Delta+B^{2} / \delta\right)}$. For further details of the derivation the reader is referred to Ref. [38]. 

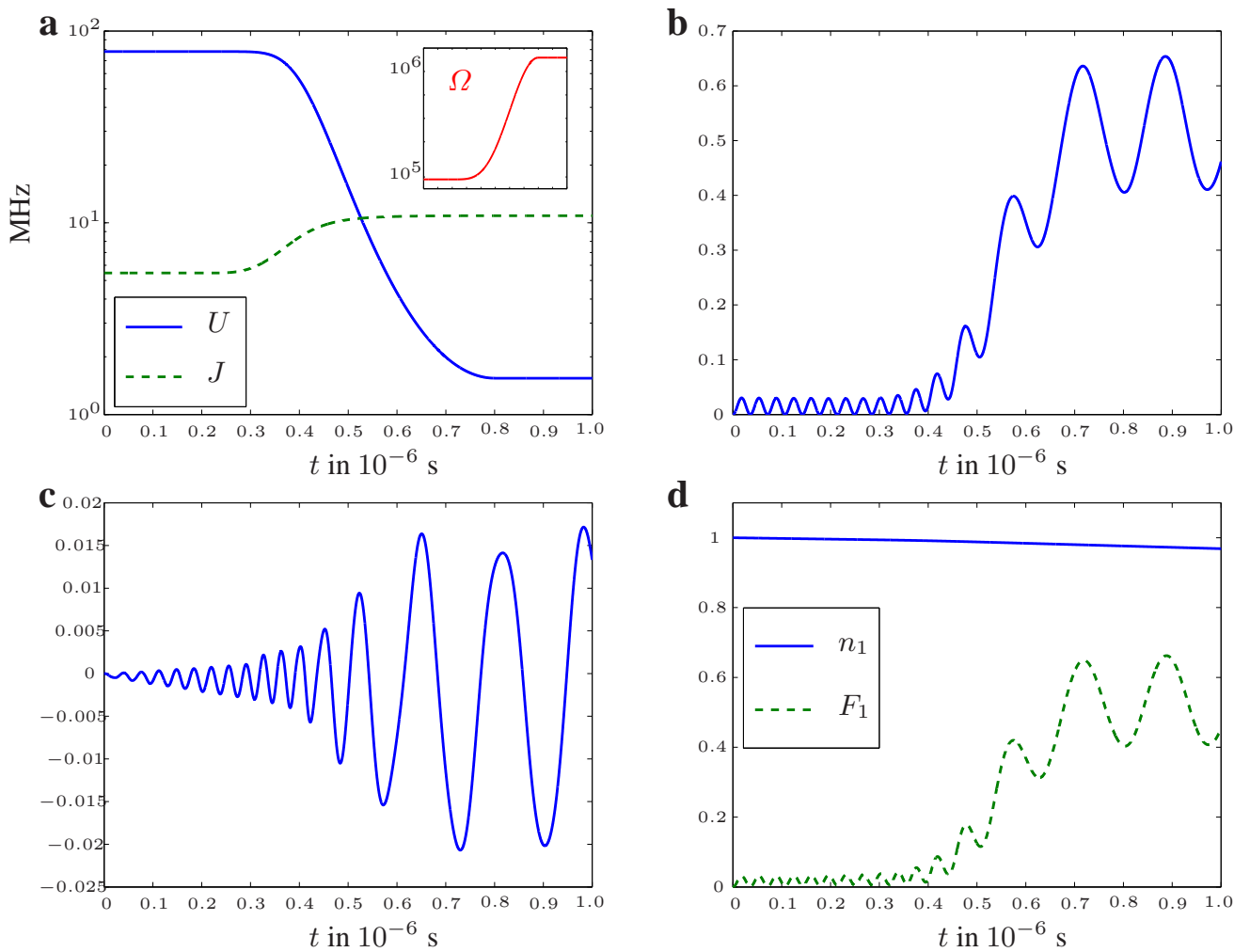

Figure 5 The Mott insulator to superfluid transition for 3 polaritons in 3 cavities compared to 3 particles in a 3 site Bose-Hubbard model. a: Log-plot of $U$ and $J$ and linear plot of the time dependent $\Omega$ (inset). b: Number fluctuations for polaritons in cavity $1, F_{1}$, for a single quantum jump trajectory. c: Difference between number fluctuations for polaritons in a cavity and number fluctuations in a pure Bose-Hubbard model, $\delta F_{1}$, for a single quantum jump trajectory. d: Expectation value and fluctuations for the number of polaritons in one cavity/site according to the effective model (7) with damping [19. $g_{24}=g_{13}=2.5 \times 10^{9} s-1, \gamma_{4}=\gamma_{3}=1.6 \times 10^{7} s^{-1}$, $\kappa=0.4 \times 10^{5} \mathrm{~s}^{-1}, N=1000, \Delta=-2.0 \times 10^{10} \mathrm{~s}^{-1}$ and $2 \omega_{C} \alpha=1.1 \times 10^{7} \mathrm{~s}^{-1}$. The Rabi frequency of the driving laser is increased from initially $\Omega=7.9 \times 10^{10} \mathrm{~s}^{-1}$ to finally $\Omega=1.1 \times 10^{12} \mathrm{~s}^{-1}$. Deviations from the pure Bose-Hubbard model are about $2 \%$. Reprinted with permission from [21].

An example how the parameters of the effective Hamiltonian (20) vary as a function of the intensity of the driving laser $\Omega$ is given in figure 6 , where the parameters of the atom cavity system are chosen to be $g_{24}=g_{13}, N=1000$, $\Delta=-g_{13} / 20, \delta=2000 \sqrt{N} g_{13}$ and $\alpha=g_{13} / 10$. Figure 6) shows the interactions $U_{b}, U_{c}$ and $U_{b c}$, the tunnelling rates $J_{b b}, J_{c c}$ and $J_{b c}$ and $\left|\mu_{c}-\mu_{b}\right|$ as a function of $\Omega / g_{13}$. For $g \approx \Omega$ one has $\left|U_{b c}\right| \ll\left|U_{b}\right|,\left|U_{c}\right|$ and $J_{b b} \approx J_{c c} \approx$ $J_{b c}$. Whenever $\left|\mu_{c}-\mu_{b}\right|<\left|J_{b c}\right|, b^{\dagger}$ polaritons get converted into $c^{\dagger}$ polaritons and vice versa via the tunnelling $J_{b c}$. With the present choice of $\alpha$ and $\delta$, this happens for $0.16 g<\Omega<1.6 g$.

\subsection{Frozen light: photonic Bose-Hubbard models}
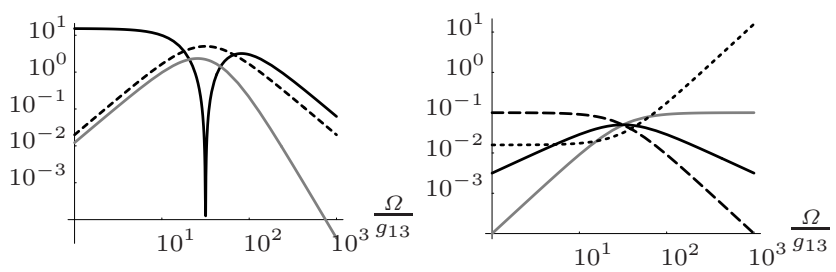

Figure 6 Left: The polariton interactions $U_{b}$ (dashed line), $U_{c}$ (grey line) and $U_{b c}$ (solid line) in units of $g_{13}$ as a function of $\Omega / g_{13}$. Right: The tunnelling rates $\left|J_{b b}\right|$ (dashed line), $\left|J_{c c}\right|$ (grey line) and $\left|J_{b c}\right|$ (solid line) together with $\left|\mu_{c}-\mu_{b}\right|$ (dotted line) in units of $g_{13}$ as a function of $\Omega / g_{13}$. The parameters of the system are $g_{24}=g_{13}, N=1000, \Delta=-g_{13} / 20$ $\delta=2000 \sqrt{N} g_{13}$ and $\alpha=g_{13} / 10$. Reprinted with permission from [38].

than unity, $\xi \gg 1$ is achieved, it is possible to engineer polaritonic Bose-Hubbard models in coupled cavity arrays.
In the last section we have discussed that, as soon as a strong coupling regime with a cooperativity factor larger 
The prospect that even higher cooperativity factors (of the order of $10^{2}-10^{3}[81]$ ) can be realised in some cavity QED setups, opens up the possibility of engineering a Bose-Hubbard model also for the cavity mode photons. Such a setting is actually conceptually simpler than the polaritonic and has already been outlined before. Given that the photonic tunnelling term is a consequence of the interaction of neighbouring cavities, to realise a photonic BoseHubbard model one needs to find a way to efficiently create large photon Kerr nonlinearities using the interaction of the atoms with the cavity mode. In this section we review two proposals to this aim.

To create a pure photonic Bose-Hubbard model would be very interesting as in this way new regimes for light, which do not occur naturally, could be engineered. For example, in the photonic Mott insulator exactly one photon exists in each cavity, provided the whole structure contains on average one photon per cavity. Moreover, the photons are localised in the cavity they are in and are not able to hop between different cavities. In such a situation photons behave as strongly correlated particles that are each "frozen" to their lattice site, a system that would correspond to a crystal formed by light.

The first proposal for generating strong Kerr photon nonlinearities, which we discuss here, is the EIT based setting analysed in the last section. As shown in [25], in the regime characterised by $g \ll \Omega$ and $g_{24} g \ll|\Delta \Omega|$, the dark sate polariton $p_{0}$ becomes a photon and the nonlinearity, c.f. equation (15), is given by

$$
U=-\frac{g_{24} g}{|\Delta \Omega|} \frac{g}{\Omega} g_{24}\left(a^{\dagger}\right)^{2} a^{2}
$$

The effective decay rate for the photons subject to the nonlinearity is given by

$$
\Gamma=\kappa+\Theta(n-2) g_{24}^{2} g^{2} \Delta^{-2} \Omega^{-2} \gamma_{4},
$$

where cavity decay is the main source of loss since $g_{24} g \ll$ $|\Delta \Omega|$.

Taking, for example, the case of toroidal micro-cavities, for which the achievable parameters are predicted to be $g_{24}=2.5 \times 10^{9} s^{-1}, \gamma_{4}=1.6 \times 10^{7} s^{-1}$, and $\kappa=0.4 \times$ $10^{5} \mathrm{~s}^{-1}$ [81], and setting the parameters such that $g / \Omega=$ $g_{24} g /|\Delta \Omega|=0.1$, leads to $U / \Gamma=625$, which would be sufficient to observe a Mott insulator of photons, as shown in figure 7

A second proposal to realise large Kerr nonlinearities is due to Ref. [26]. There a new configuration, involving a three levels lambda structure and only one coupling to the cavity mode (see figure 8), was shown to produce nonlinearities as large as the EIT based setting discussed before. A possible advantage of this approach is that, as the only three levels are used, it might be easier to implement it in some of the new cavity QED systems, such as quantum dots in photonic crystal cavities or Cooper pair boxes in stripline cavities.

The relevant atomic level structure, depicted in figure 8 is a $\Lambda$ system with two metastable states and an excited
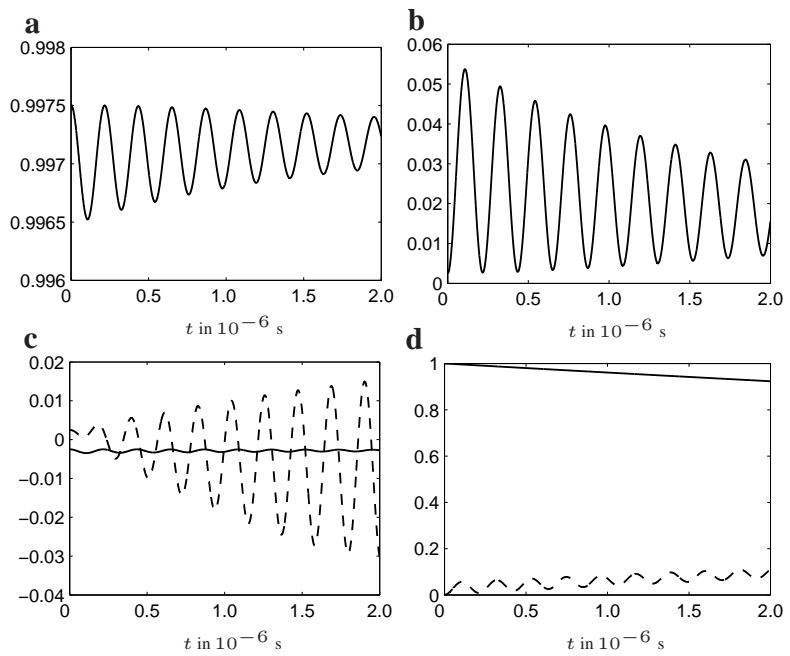

Figure 7 Mott insulator state for three photons in three cavities: a: photon number $n_{1}$, b: photon number fluctuation $F_{1}$, c: differences between full and effective Bose-Hubbard model, $\delta n_{1}$ (solid line) and $\delta F_{1}$ (dashed line) and $\mathbf{d}: n_{1}$ and $F_{1}$ as given by a solution of a master equation for the three site Bose-Hubbard model (7) including damping $\Gamma$. Reprinted with permission from [25.

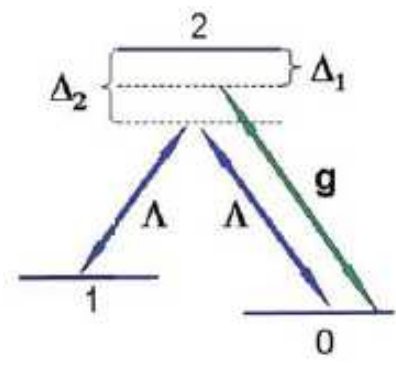

Figure 8 The level structure for the generation of the effective Hamiltonian 26. $\left(g, \Delta_{1}\right)$ and $\left(\Lambda, \Delta_{2}\right)$ are the Rabi frequencies and detunings of the cavity-atom and laser-atom interactions. Reprinted with permission from [26].

state. The cavity mode couples dispersively only to the $0-$ 2 transition and the levels 0 and 1 are coupled via a fardetuned Raman transition. Finally, the detuning associated with the lasers and with the cavity mode are assumed to be sufficiently different from each other. The origin of the nonlinearity can be understood entirely by considering the Stark shifts due to the dispersive interactions.

The full Hamiltonian of the system, in the interaction picture, reads

$$
H=g e^{-i \Delta_{1} t} a S_{20}+\sqrt{2} \Lambda e^{-i \Delta_{2} t} S_{2+}+\text { h.c. }
$$

where $S_{20}:=\sum_{k}\left|2_{k}\right\rangle\left\langle 0_{k}\right|$ and $S_{2+}:=\sum_{k}\left|2_{k}\right\rangle\left\langle+_{k}\right|$. As shown in figure $8,\left(g, \Delta_{1}\right)$ and $\left(\Lambda, \Delta_{2}\right)$ are the Rabi frequencies and detunings of the cavity-atom and laser-atom interactions, respectively. In the dispersive regime, characterised by $\frac{\sqrt{N} g}{\Delta_{1}} \ll 1$ and $\frac{\sqrt{N} \Lambda}{\Delta_{2}} \ll 1$, where furthermore $\sqrt{N} g, \sqrt{N} \Lambda \ll\left|\Delta_{2}-\Delta_{1}\right|$, the processes driven by the cavity-atom and laser-atom interactions can be treated in- 
dependently and the excited state will hardly be populated. Hence an effective Hamiltonian for the two metastable states and the cavity mode can be found by adiabatically eliminating the excited state. Dropping out terms proportional to the identity, it is given by

$$
H_{1}=\frac{g^{2}}{\Delta_{1}} a^{\dagger} a S_{00}+\frac{\Theta}{2}\left(S_{10}+S_{01}\right),
$$

with $\Theta:=2 \Lambda^{2} / \Delta_{2}$.

In a second interaction picture with respect to $H_{0}=$ $\frac{g^{2}}{2 \Delta_{1}} a^{\dagger} a$ it reads $H_{1}^{i n t}=\frac{g^{2}}{2 \Delta_{1}} a^{\dagger} a\left(S_{+-}+S_{-+}\right)+\frac{\Theta}{2} S_{3}$, where $S_{+-}:=\sum_{k}\left|+_{k}\right\rangle\left\langle-_{k}\right|, S_{-+}=\left(S_{+-}\right)^{\dagger}$, and, as before, $S_{3}:=\sum_{k=1}^{N}\left|+_{k}\right\rangle\left\langle+_{k}|-|-_{k}\right\rangle\left\langle-_{k}\right|$. In the $\{| \pm\rangle\}$ basis the system can thus be viewed as an ensemble of two level atoms driven by a laser with a photon-numberdependent Rabi frequency. Considering the dispersive regime of this system, $\frac{\sqrt{N} g^{2}}{2 \Delta_{1} \Theta} \ll 1$, the atoms prepared in the $|-\rangle$ state will experience a Stark shift proportional to $\left(a^{\dagger} a\right)^{2}$, which gives rise to a strong Kerr nonlinearity. The effective Hamiltonian is be given by $H_{\text {eff }}=\frac{g^{4}}{4 \Delta_{1}^{2} \Theta} a^{\dagger} a a^{\dagger} a S_{3}$. Therefore, if all atoms are prepared in the $|-\rangle$ state, one obtains an essentially absorption free Kerr nonlinearity given by

$$
H_{k e r r}=\frac{\sqrt{N} g^{2}}{2 \Delta_{1} \Theta} \frac{\sqrt{N} g}{2 \Delta_{1}} g a^{\dagger} a a^{\dagger} a .
$$

\subsection{Measurements}

In this section we discuss ways how information about the state of the polaritonic Bose-Hubbard models can be obtained in experiments. The possibilities to access the system in measurements are somewhat complementary to those for cold atoms in optical lattices [8]. Whereas time of flight images give access to global quantities such as phase coherence in optical lattices, coupled cavity arrays allow to access the local particle statistics in measurements. Unlike optical lattices they would thus allow to obtain direct experimental evidence for the exact integer particle number in each lattice site in a Mott Insulator regime. We first discuss the measurement scheme for the one-component model and then turn to its extension to for the two-component model.

\subsubsection{One-component model}

For the model (7), the number of polaritons in one individual cavity can be measured via state selective resonance fluorescence. To that end, the polaritons are transformed into purely atomic excitations by adiabatically switching off the driving laser. The process is adiabatic if $g B^{-2} \frac{d}{d t} \Omega \ll$ $\left|\mu_{+}\right|,\left|\mu_{-}\right|$[76], which means it can be fast enough to prevent polaritons from hopping between cavities during the switching. Hence, in each cavity, the final number of atomic excitations in level 2 is equal to the initial number of dark state polaritons, while the other polariton species will not be transferred to the atomic level 2 (c.f. (10). In this state cavity loss is eliminated and the atomic excitations are very long lived. They can then be measured with high efficiency and precision using state-selective resonance fluorescence as proposed in [86] and [87].

\subsubsection{Two-component model}

The number statistics for both polariton species for the model (20), $b^{\dagger}$ and $c^{\dagger}$, in one cavity can again be measured using state selective resonance fluorescence. In the two-component case the STIRAP can however not be applied as in the single component case because the energies $\mu_{b}$ and $\mu_{c}$ are similar and the passage would thus need to be extremely slow to be adiabatic.

For two components, one can do the measurements as follows. First the external driving laser $\Omega$ is switched off. Then the roles of atomic levels 1 and 2 are interchanged in each atom via a Raman transition by applying a $\pi / 2$-pulse. To this end the transitions $1 \leftrightarrow 3$ and $2 \leftrightarrow 3$ are driven with two lasers (both have the same Rabi frequency $\Lambda$ ) in two-photon resonance for a time $T=\pi \delta_{\Lambda} /|\Lambda|^{2}\left(\delta_{\Lambda}\right.$ is the detuning from atomic level 3 ). The configuration is shown in figure 9. This pulse results in the mapping $\left|1_{j}\right\rangle \leftrightarrow\left|2_{j}\right\rangle$ for all atoms $j$.

Next another laser, $\Theta$, that drives the transition $1 \leftrightarrow 4$ is switched on, see figure $9 \mathrm{~b}$. Together with the coupling $g_{24}$, this configuration can be described in terms of three polaritons, $q_{0}^{\dagger}, q_{+}^{\dagger}$ and $q_{-}^{\dagger}$, in an analogous way to $p_{0}^{\dagger}, p_{+}^{\dagger}$ and $p_{-}^{\dagger}$, where now the roles of the atomic levels 1 and 2 and the levels 3 and 4 are interchanged. Hence, if one chooses $\Theta=\Omega$ the $\pi / 2$-pulse maps the $b^{\dagger}$ onto the dark state polaritons of the new configuration, $q_{0}^{\dagger}$, whereas for $\Theta=-\Omega$ it maps the $c^{\dagger}$ onto $q_{0}^{\dagger}$. The driving laser is then adiabatically switched off, $\Theta \rightarrow 0$, and the corresponding STIRAP process maps the $q_{0}^{\dagger}$ completely onto atomic excitations of level 1 . This process can be fast since the detuning $\Delta$ is significantly smaller than $\delta$ and hence the energies of all polariton species $q_{0}^{\dagger}, q_{+}^{\dagger}$ and $q_{-}^{\dagger}$ are well separated. Another $\pi / 2$-pulse finally maps the excitations of level 1 onto excitations of level 2, which can be measured by state selective resonance fluorescence in the same way as for the one-component model.

The whole sequence of $\pi / 2$-pulse, STIRAP process and another $\pi / 2$-pulse can be done much faster than the timescale set by the dynamics of the Hamiltonian (20) and $b^{\dagger}$ or $c^{\dagger}$ can be mapped onto atomic excitations in a time in which they are not able to move between sites. The procedure thus allows to measure the instantaneous local particle statistics of each species separately. 

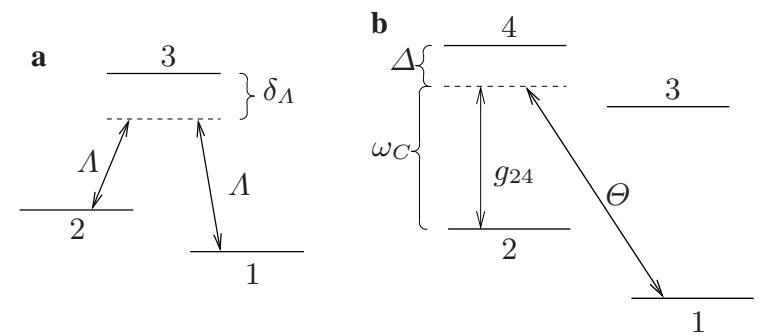

Figure 9 a: Configuration of the $\pi / 2$-pulse. Two driving lasers in two-photon transition with identical Rabi frequencies $\Lambda$ couple to the atomic transitions $1 \leftrightarrow 3$ and $2 \leftrightarrow 3$. b: Configuration for the STIRAP process. A driving laser couples to the $1 \leftrightarrow 4$ transition with Rabi frequency $\Theta$. The cavity mode couples to transitions $2 \leftrightarrow 4$ and $1 \leftrightarrow 3$, where the coupling to $1 \leftrightarrow 3$ is ineffective and not shown. Reprinted with permission from [38].

\section{Models Based on the Jaynes-Cummings Hamiltonian}

Many-body dynamics on a lattice that can either be dominated by the tunnelling between adjacent lattice sites or by an on-site interaction can also be generated in an array of coupled cavities with Jaynes-Cummings type coupling between cavity photons and two level atoms in each cavity. As shown in [22] and [23], the anharmonicity of the energy levels of the dressed states of a Jaynes-Cummings Hamiltonian acts as an effective on-site interaction. To see this let us recall the Jaynes-Cummings Hamiltonian for one atom,

$$
H^{J C}=\omega_{C} a^{\dagger} a+\omega_{0}|e\rangle\langle e|+g\left(a^{\dagger}|g\rangle\langle e|+a| e\rangle\langle g|\right),
$$

where $\omega_{C}, \omega_{0}$ are the frequencies of the resonant mode of the cavity and of the atomic transition, respectively, $g$ is Jaynes-Cummings coupling between the cavity mode and the two level system, $a^{\dagger}$ is the creation operator of a photon in the resonant cavity mode, and $|g\rangle,|e\rangle$ are the ground and excited states of the two level system.

Since the energy of a photon $\omega_{C}$ and the atomic transition energy $\omega_{0}$ are much greater than the atom photon coupling $g$, the number of excitations is conserved for the Hamiltonian (27). Hence it can be diagonalised for each manifold with a fixed number of excitations $n$ separately. The energy eigenvalues for $n$ excitations read $E_{0}=0$ and

$$
E_{n}^{ \pm}=n \omega_{C}+\frac{\Delta}{2} \pm \sqrt{n g^{2}+\frac{\Delta^{2}}{4}}
$$

for $n \geq 1$, where $\Delta=\omega_{0}-\omega_{C}$.

Let us now consider an array of cavities where the atom photon interaction in each cavity is described by equation (27) and assume that we have prepared the system in a state with one excitation of energy $E_{1}^{-}$in each cavity. Since the lowest energy for two excitations in one cavity is $E_{2}^{-}$, moving one additional excitation to a cavity requires an extra energy of

$$
E_{2}^{-}-2 E_{1}^{-}=2 \sqrt{g^{2}+\frac{\Delta^{2}}{4}}-\sqrt{2 g^{2}+\frac{\Delta^{2}}{4}}-\frac{\Delta}{2},
$$

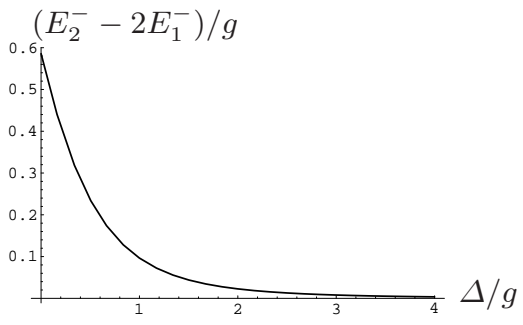

Figure 10 Dependence of the effective repulsion $E_{2}^{-}-2 E_{1}^{-}$on the detuning $\Delta$. The effective repulsion vanishes for large $\Delta$

which plays the role of an effective on-site repulsion. This repulsion can be tuned via the detuning $\Delta$ as figure 10 shows.

The full effective many-body model in a cavity array that is based on the effective interaction (29) reads,

$$
H=\sum_{\mathbf{R}} H_{\mathbf{R}}^{J C}-J \sum_{<\mathbf{R}, \mathbf{R}^{\prime}>}\left(a_{\mathbf{R}^{\dagger}}^{\dagger} a_{\mathbf{R}^{\prime}}+\text { h.c. }\right),
$$

where $\mathbf{R}$ labels the site of a cavity. Each cavity contains one atom interacting via the Jaynes-Cummings interaction with the cavity mode and photons tunnel between neighbouring cavities at a rate $J$.

Figure 11 gives evidence for the Mott insulator and superfluid regimes of Hamiltonian (30). The probabilities to find one or two excitations in one cavity are shown for an array where initially one cavity had no and all others one excitation. For detuning $\Delta=0$, double occupations never occur, indicating a Mott insulator behaviour. For detunings $\Delta \gg g$, on the other hand, occupation numbers larger than one occur as to be expected for a superfluid regime. In the deep Mott insulator regime, the model (30) can also reproduce effective spin models [?], see section 7.1

Effective many-body physics based on the Hamiltonian (30) can not only be observed for a single two level system in each cavity, as assumed in (27), but also for setups with several two level systems per cavity. Such a model can describe photonic crystal micro-cavities doped with substitutional donor or acceptor impurities. This approach to implementing effective many-body models, which can have suitable parameters, has been proposed in [44]. The phase transitions of a model with several two level systems in each cavity have also been studied in [39 47].

To allow for the observation of coherent effective manybody dynamics, the effective on-site repulsion (29) has to be much stronger than the rates of spontaneous emission and cavity decay. Since the dressed states are superpositions of a photonic and an atomic component, they are vulnerable to both decay mechanisms. Spontaneous emission can be suppressed by increasing the detuning $\Delta$ as this causes the occupation of the excited atomic level to decrease. An increase of $\Delta$ however also causes the repulsion to decrease as shown in figure 10. The effective many-body dynamics is thus only observable if each cavity operates in the strong coupling regime, see section 2 . 


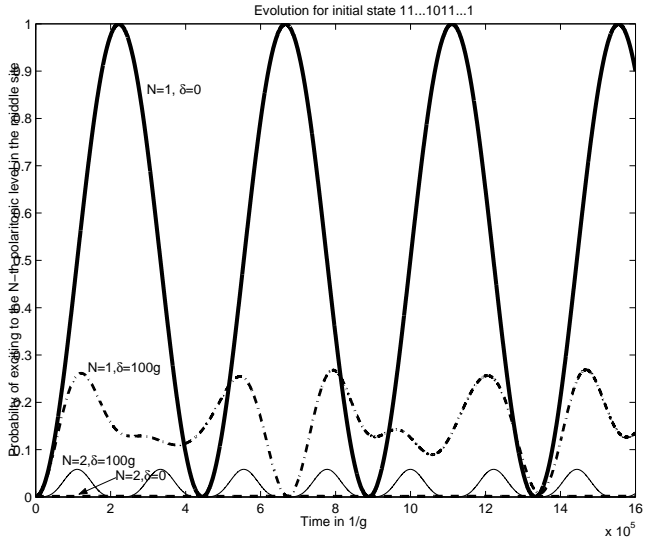

Figure 11 Probabilities for finding one (thick solid line) or two (thick dashed line) excitations in the middle cavity which was initially taken to be empty (in the resonant regime). In this case, as there were initially fewer excitations than cavities, oscillations occur for the single excitation. The double one is never occupied due to the blockade effect-Mott insulator phase (thick dashed line). For the detuned case however, hopping of more than one excitation is allowed (superfluid regime) which is evident as the higher excitation manifolds are being populated now (dashed line and thin solid line). Reprinted with permission from [22].

As for the polaritonic Bose-Hubbard model (section 4.1), the transition between Mott insulator and superfluid phase can be characterised by the fluctuations of the number of excitations in one single cavity. Here, the number of excitations in cavity $l$ is measured by the operator $\mathcal{N}_{l}=$ $a_{l}^{\dagger} a_{l}+\left|e_{l}\right\rangle\left\langle e_{l}\right|$ and their fluctuations $F_{l}$ are given by the ground state variance of $\mathcal{N}_{l}, F_{l}=\left\langle\mathcal{N}_{l}^{2}\right\rangle-\left\langle\mathcal{N}_{l}\right\rangle^{2}$. Figure 12 shows the number fluctuations $F_{l}$, which play the role of an order parameter, as a function of the atom cavity detuning $\Delta$. Whereas the fluctuations are very small in the Mott insulator regime (small detuning), they become significantly larger in the superfluid phase (large detuning).

Various approaches have shown that this model indeed exhibits a quantum phase transition that closely resembles the Mott insulator to superfluid transition of the BoseHubbard model [22, 23, 39, 44, 40, 42], see also section 6. On the other hand deviations of the phase transitions in the model (30) from the Mott insulator to superfluid transition in a Bose-Hubbard model have also been found.

In this analysis exact diagonalisations of small models of very few cavities have been performed [41]. Here, insulator states have been found, where the number of excitations in one site does not fluctuate and the effective particles are either atomic (for $\Delta / g<-1$ and $|J|<|\Delta|$ ) or polaritonic $(\Delta / g \lesssim 1$ and $|J| \lesssim|\Delta|)$. Superfluid states on the other hand show local excitation number fluctuations and can either contain polaritonic $(\Delta / g<-1$ and $|J| \approx|\Delta|)$ or photonic $(\Delta<0,|J|>|\Delta| \gtrsim g$ or $\Delta>$ $0,|J| \gtrsim g$ ) effective particles.

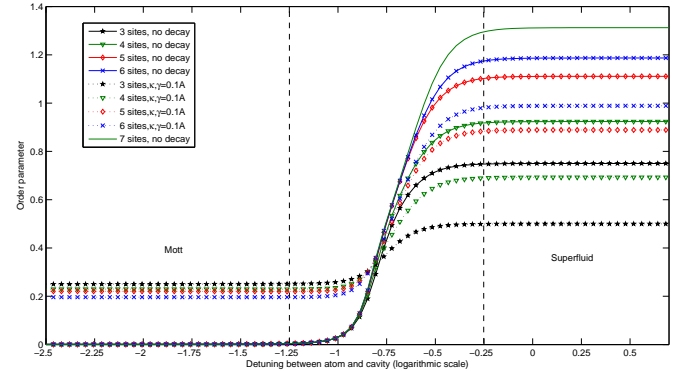

Figure 12 The order parameter $F_{l}$ as a function of the detuning between the hopping photon and the doped two-level system in logarithmic units of the matter-light coupling $g$. Simulations include results for 37 sites, with and without dissipation due to spontaneous emission and cavity leakage. The graphs are obtained by diagonalisation of the Hamiltonian 30 .

For the characterisation of the various phases, possible signatures in the visibility of interference fringes of photons that could be emitted by the cavities have been discussed [39,50]. The visibility $\mathcal{V}$ of the interference pattern is given in terms of the photon number distribution in momentum space, $S(k)=\frac{1}{L} \sum_{j, l=1}^{L} \mathrm{e}^{2 \pi \mathrm{i}(j-l) / L}\left\langle a_{j}^{\dagger} a_{l}\right\rangle,(L$ is the number of sites in a one dimensional array) by $\mathcal{V}=$ $(\max (S)-\min (S)) /(\max (S)+\min (S))$. In the superfluid regime, $\mathcal{V}$ approaches unity whereas it is very small for a Mott insulator.

\section{Phase Diagrams}

As already indicated above one of the key questions for effective many-body systems in coupled cavity arrays is which quantum phase transitions these can show. The phase diagram of both, polaritonic Bose-Hubbard models and Jaynes-Cummings interaction based models, has been analysed in various settings. Approaches based on different techniques have been carried out for one- and two-dimensional models.

The phase boundary between a Mott insulator and a superfluid phase can be determined in a grand canonical approach. To this end, a chemical potential $\mu$ is introduced and the grand canonical Hamiltonian reads,

$$
H^{G C}=H-\mu \mathcal{N},
$$

where the operator $\mathcal{N}$ counts the total number of excitations and is given by

$$
\mathcal{N}=\sum_{\mathbf{R}}\left(a_{\mathbf{R}}^{\dagger} a_{\mathbf{R}}+\left|e_{\mathbf{R}}\right\rangle\left\langle e_{\mathbf{R}}\right|\right)
$$

for Jaynes-Cummings interaction based models (30) and

$$
\mathcal{N}=\sum_{\mathbf{R}}\left(a_{\mathbf{R}}^{\dagger} a_{\mathbf{R}}+\left|2_{\mathbf{R}}\right\rangle\left\langle 2_{\mathbf{R}}|+| 3_{\mathbf{R}}\right\rangle\left\langle 3_{\mathbf{R}}|+2| 4_{\mathbf{R}}\right\rangle\left\langle 4_{\mathbf{R}}\right|\right)
$$


for EIT based models (9), which lead to polaritonic BoseHubbard models. Here, an excitation of the atomic level 4 is equivalent to two polariton excitations since an atom needs to absorb two cavity photons to make a transition from level 1 to level 4, whereas the transition from level 1 to 2 or 3 only requires one cavity photon. The occupations of level 4 thus carry a double weight.

We note that the chemical potential $\mu$ introduced in equation (31) is conceptually different from the chemical potential $\varepsilon$ in equation (16). The approach in section 4.1 considers a closed system without particle exchange with the environment and $\varepsilon$ is an energy shift that affects the polaritons. The grand canonical approach in this section, in contrast, considers a situation in which particle exchange with the surrounding is permitted and is used because of its convenience for determining the phase diagrams.

The total number of excitations is conserved for both models, $[H, \mathcal{N}]=0$. The boundary between Mott insulator and superfluid phases is determined by the value of $\mu$ for which adding or removing a particle does not require energy.

\subsection{Phase diagrams for one dimensional models}

The phase diagrams for the one dimensional versions of both, polaritonic Bose-Hubbard and Jaynes-Cummings interaction based models, have been obtained by DMRG calculations (see [88] for a review on DMRG). These results were presented in [39], where in addition the existence of a polaritonic glassy phase in setups with disorder has been predicted.

The authors consider a linear array of $L$ cavities with various numbers of atoms, $N$, in each cavity. Figure 13 shows the phase diagram and compressibility for the model (30). Here $\omega$ denotes the frequency of the cavity-photons $\left(\omega \equiv \omega_{C}\right), \epsilon$ the energy of the excited atomic levels $(\epsilon \equiv$ $\left.\omega_{0}\right), \beta$ the atom-photon coupling $(\beta \equiv g)$ and $\rho$ the number of excitations per cavity. The compressibility $\kappa$ is defined by $\kappa=\partial \rho / \partial \mu$. The Mott insulator is characterised by a vanishing compressibility whereas $\kappa$ is finite for a superfluid. Figure 14 shows the phase diagram and compressibility for the model (9).

For a Bose-Hubbard Hamiltonian, the lobe width at $t=0$ is given by the on-site repulsion $U$ and the critical value $t^{*}$ of the hopping strength at which the various lobes shrink into a point is proportional to $U$. In contrast to the Bose-Hubbard model, in both models, 30) and (9), for fixed $N$, the critical values $t^{*}$ are not proportional to the lobe width at $t=0$. Furthermore, the ratio between the upper and the lower slopes of the lobes at small hopping is greater than the one predicted for the Bose-Hubbard model $\left(2 n_{\text {polariton }}\right.$ for the lower and $-2\left(n_{\text {polariton }}+1\right)$ for the upper slope [89]); this discrepancy disappears on increasing the number of atoms inside the cavity. In terms of an effective Bose-Hubbard model, this may be interpreted as
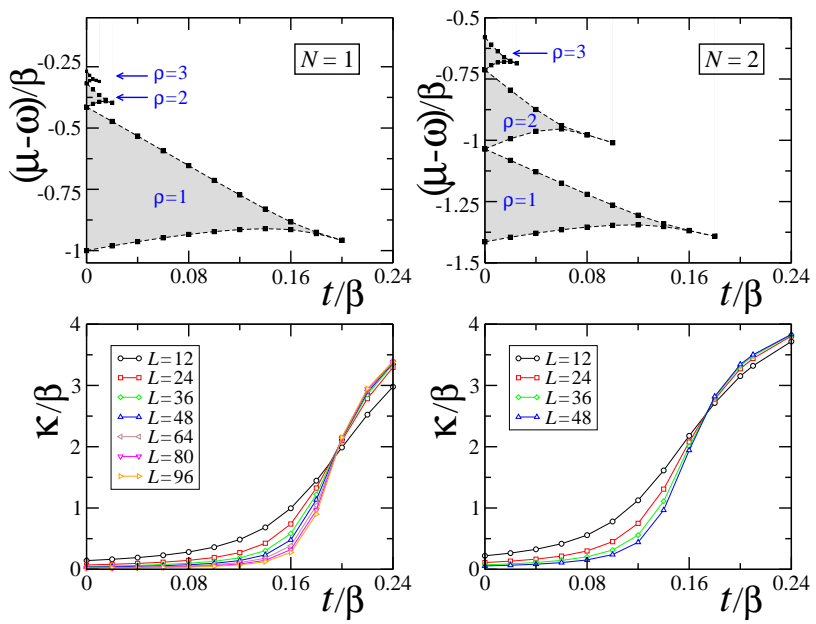

Figure 13 Upper panels: Phase diagram for the Hamiltonian model 30 , with $N=1,2$ atoms inside each cavity at $\epsilon=\omega$. Lower panels: System compressibility $\kappa$ for the first lobe (i.e., $\rho=1$ ), for different system sizes $L$, with $N=1$ (left) and $N=2$ (right). Reprinted with permission from [39].
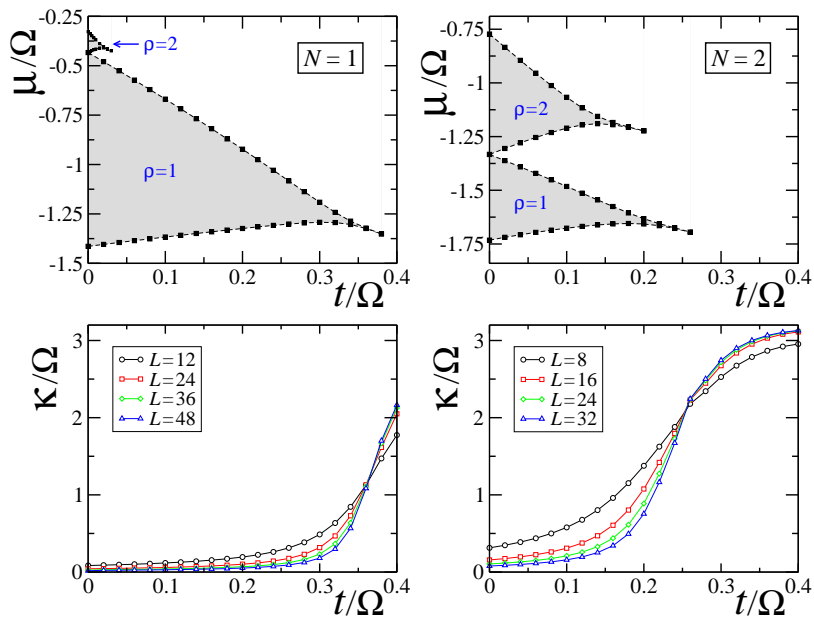

Figure 14 Upper panels: Phase diagram of model 9. The detuning parameters are set to zero and $g / \Omega=1$. Lower panels: System compressibility $\kappa$ for the first lobe, for different system sizes $L$, with $N=1$ (left) and $N=2$ (right). Reprinted with permission from [39].

a correlated hopping of the polaritons, where the hopping depends on the occupation of the cavity. For larger occupations, the polaritons have a stronger tendency to tunnel between cavities and consequently the superfluid regime is already reached for lower values of the hopping term prefactor $t$. Deviations of the critical hopping $t^{*}$ from the critical hopping in a Bose-Hubbard model vanish for $N \gtrsim 10$ atoms per cavity [39]. 
For the detection of the Mott insulator-superfluid quantum phase transition, the polariton and photon fluctuations in a block of linear dimension $M$ (in units of the lattice constant of the array) have been studied [49]. Whereas polariton fluctuations are independent of the block size $M$ in the Mott insulator regime, they grow logarithmically with $M$ in the superfluid phase. The prefactor of the logarithm is thereby related to the compressibility of the system. For the photon fluctuations, the scaling of the leading terms is always linear in $M$ and non-critical while the critical behaviour is encoded in the subleading scaling [49].

\subsection{Phase diagrams for two dimensional models}

The phase diagrams for the two dimensional version of the Jaynes-Cummings based model (30) have been calculated by various approaches, among those a mean field approach [23] and a variational cluster approach [40].

\subsubsection{Mean-field phase diagram for the $2 \mathrm{~d}$}

Jaynes-Cummings based model

A mean-field phase diagram for the two dimensional version of the model (30) on a triangular lattice has been obtained in [23]. Introducing the superfluid order parameter $\Psi=\left\langle a_{\mathbf{R}}\right\rangle$ and using the decoupling approximation $a_{\mathbf{R}}^{\dagger} a_{\mathbf{R}^{\prime}}=\left\langle a_{\mathbf{R}}^{\dagger}\right\rangle a_{\mathbf{R}^{\prime}}+a_{\mathbf{R}}^{\dagger}\left\langle a_{\mathbf{R}^{\prime}}\right\rangle+\left\langle a_{\mathbf{R}}^{\dagger}\right\rangle\left\langle a_{\mathbf{R}^{\prime}}\right\rangle$, one obtains the mean-field Hamiltonian,

$$
\begin{aligned}
H^{M F}= & \sum_{\mathbf{R}}\left[H_{\mathbf{R}}^{J C}-z J \Psi\left(a_{\mathbf{R}}^{\dagger}+a_{\mathbf{R}}\right)+z J|\Psi|^{2}\right. \\
& \left.-\mu\left(a_{\mathbf{R}}^{\dagger} a_{\mathbf{R}}+\left|e_{\mathbf{R}}\right\rangle\left\langle e_{\mathbf{R}}\right|\right)\right]
\end{aligned}
$$

where $z$ counts the number of nearest neighbours and is here set to $z=3$ since a triangular lattice is assumed [23]. Here again, a chemical potential $\mu$ has been introduced in (34) and the phase boundary between superfluid and Mott insulator is given by the value of $\mu$, for which adding or removing one excitation to or from the system does not cost energy.

The mean-field phase diagram for the two dimensional model (34) on a triangular lattice is shown in figure 15. Here, $\Psi=0$ corresponds to stable Mott lobes, where the number of excitations in each cavity increases with $\mu$. The regions on the right correspond to $\Psi \neq 0$, where the system will be found in a superfluid phase, i.e. within the mean field approximation, the ground state corresponds to a coherent state of photons in each cavity. The number of photons in each Mott lobe can be confirmed by considering the average number of excitations per site in the grand canonical ensemble $\rho=\partial E_{g} / \partial \mu$, where $E_{g}$ is the ground state energy. Figure 16 shows $\rho$ as a function of the hopping and chemical potential. The plateaus indicate regions
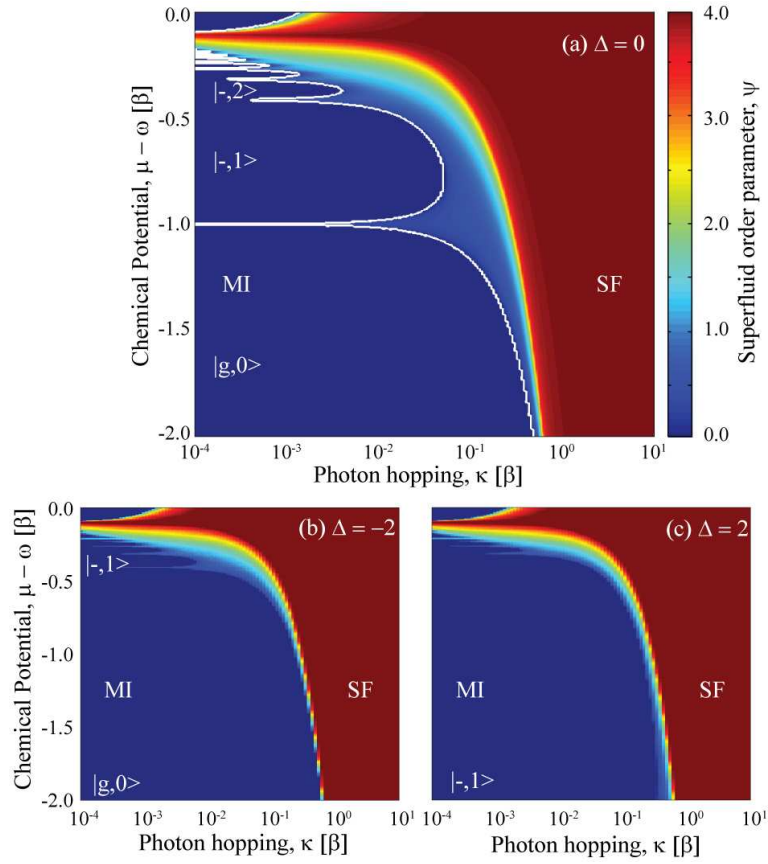

Figure 15 Slices showing the superfluid order parameter, $\Psi$, as a function of the photon hopping frequency, here denoted by $\kappa$ $(\kappa \equiv J)$, and the chemical potential, $\mu$. For detuning, $\Delta=0$ (a), $\Delta=2 \beta$ ( $\beta$ denotes the Jaynes-Cummings coupling, $\beta \equiv g$ ) (b) and $\Delta=-2 \beta$ (c) (Note that the figure defines $\Delta=\omega_{C}-\omega_{0}$ and denotes the cavity resonance by $\omega$, contrary to the text). Mott insulator lobes are indicated by the regions of $\Psi=0$, the lowest of which are shown. Dominating the left-hand edge (where photonic repulsion dominates over hopping) is the Mott insulator phase (denoted MI), and the superfluid phase is found on the right-hand edge (denoted SF). The white contour in a corresponds to the region where $\Psi$ becomes non-zero, delineating the quantum phase transition. Reprinted with permission from [23].

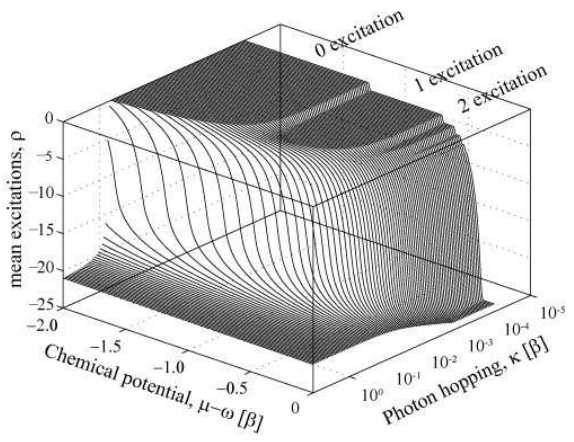

Figure 16 Plateaus with constant density, $\rho$, indicating regions with a definite number state excitations, as a function of the chemical potential $\mu$ and photon hopping frequency $\kappa$ for detuning $\Delta=0$. Regions with varying $\rho$ have coherent states as the ground state configuration. Reprinted with permission from [23]. 


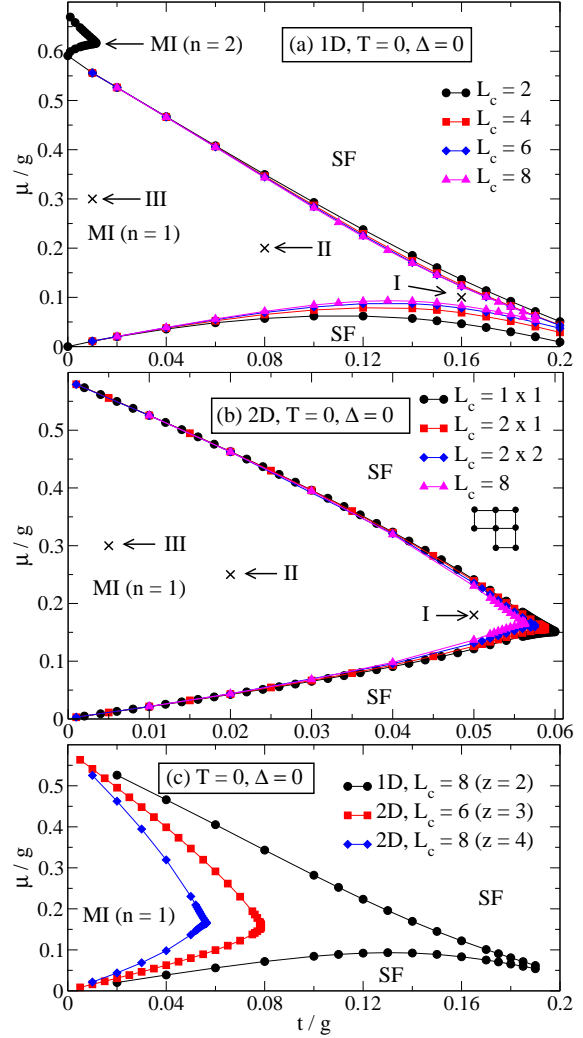

Figure 17 Ground-state phase diagram for the Hamiltonian 30: (a) 1D, (b) 2D and (c) different coordination numbers $z$. Lines are guides to the eye. Reprinted with permission from [40].

of constant density and incompressibility which are characteristic for a Mott insulator phase.

A mean-field phase diagram for settings with more than one two level system in each cavity has been calculated in [44], where 8 two level systems par cavity have been considered. Such a model describes photonic crystal microcavities doped with substitutional donor or acceptor impurities.

6.2.2. Phase diagrams for $2 \mathrm{~d}$ models from a variational cluster approach

The phase diagram of model (30) for the one and two dimensional case and the stability of its Mott insulator states with respect to non-zero temperatures have been analysed in [40]. The authors employ a variational cluster approach that is expected to become exact with increasing cluster size $L_{c}$. Figure 17 shows the phase diagram in one dimension, two dimensions and for different coordination numbers $z . \mu$ is the chemical potential, $g$ the Jaynes-Cummings coupling and $t$ the tunnelling rate of photons between neighbouring cavities. Figure 18 shows the unit filling Mott lobes for both, the one and two dimensional model (30) at finite temperatures $T$.

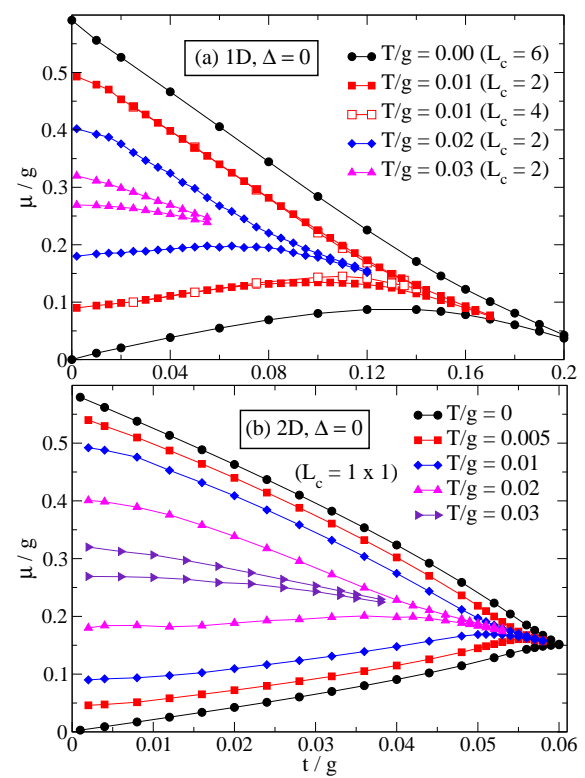

Figure 18 Mott-like regions for different $T$ for the Hamiltonian [30. Mott lobes shrink with increasing temperature $T$. Reprinted with permission from [40].

Phase diagrams for the model (30) in two dimensions have also been obtained by the stochastic series expansion quantum Monte Carlo method [43], where it has been predicted that the universality class of coupled cavity arrays may be different from the Bose-Hubbard model.

\subsection{Bose-glass phases}

A glassy phase for polaritons in a one dimensional cavity array with disorder has been predicted in [39]. This phase is characterised by a finite compressibility, gapless excitation spectrum and zero superfluid density and has been predicted to exist in a Bose-Hubbard model for relative fluctuations of the interaction, $\delta U_{\text {eff }} /\left\langle U_{\text {eff }}\right\rangle$, uniformly distributed in an interval of length $2 \epsilon=0.5$ in a system of $L=200$ sites at filling $\rho=1.01$ for $0.078 \lesssim t_{\text {eff }} /\left\langle U_{\text {eff }}\right\rangle \lesssim$ 0.133 . Figure 19 shows relative fluctuations of the interaction $\delta U_{\text {eff }} /\left\langle U_{\text {eff }}\right\rangle$ as a function of atom number fluctuations over an interval $\delta N$ in the cavities. For the model (30), a glassy phase should be visible for , e.g. $\langle N\rangle=100$ particles per cavity and $\delta N \simeq 19$ (such to have $\epsilon=0.25$ ) in the range $7.51 \times 10^{-3} \lesssim t / \beta \lesssim 1.401 \times 10^{-2}$.

The observability of quantum phase transitions from a superfluid to a Bose-glass to a Mott insulator that is driven by an decrease of the photon tunnelling rate $J$ has also been predicted for a two dimensional model in [44]. Here, photonic crystal micro-cavities doped with substitutional donor or acceptor impurities have been considered, where fluctuations in the number of impurities $(\Delta N)$, the impurity-photon coupling $\left(\Delta g_{k}\right)$ and the detunings be- 


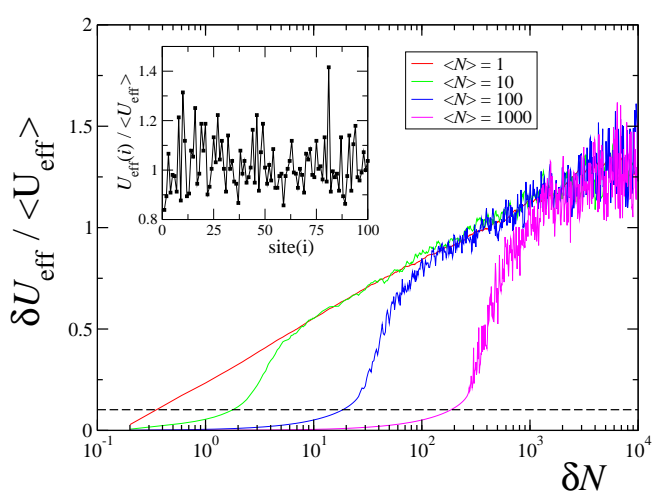

Figure 19 Relative standard deviation of the effective on-site interaction strength $U_{\text {eff }}$ averaged over $L=10^{4}$ cavities, as a function of the atom number fluctuations $\delta N$; from left to right $\langle N\rangle=1,10,100,1000$. Dashed line indicates an effective variation of the interaction strength equal to the standard deviation of a random interaction uniformly distributed in the interval $U_{\text {eff }}(i) /\left\langle U_{\text {eff }}\right\rangle \in[-\epsilon,+\epsilon]$ with $\epsilon=0.25$. In the inset an example of the variation on the on-site effective interaction is shown; $\langle N\rangle=100, \delta N=20$. Reprinted with permission from [39].

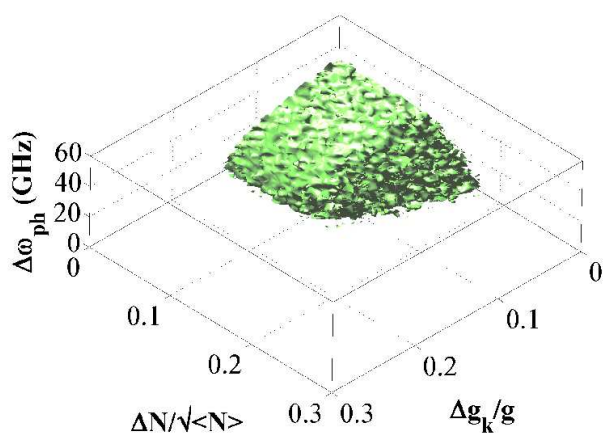

Figure 20 The isovalue surface that separates the existence and nonexistence of an equilibrium polaritonic quantum phase transition from the Bose glass to Mott insulator states. Reprinted with permission from [44].

tween impurity and photon resonances $\left(\Delta \omega_{\mathrm{ph}}\right)$ are expected in experimental realisations. The information whether an equilibrium polaritonic quantum phase transition from a Bose glass to Mott insulator states exists is obtained by plotting the isosurface in which the critical polariton tunnelling rate at the Bose glass Mott insulator phase boundary is set to be 10 times larger than the polariton loss rate, assuming a cavity Q-factor $10^{6}$, an impurity number $N=$ 3 and an impurity photon detuning $\Delta=12 g$. Inside the isovalue surface, quantum phase transitions from a superfluid to a Bose glass to Mott insulator states can be observed by continuously decreasing the tunnelling rate $J$.
Outside the isovalue surface, only superfluid and Bose glass phases exist.

\section{Effective Spin Models in Coupled Cavity Arrays}

In the previous sections we have seen that under appropriate conditions interesting bosonic many-body Hamiltonians can be created and probed in coupled cavity arrays. There the atoms were used for detection and most importantly to generate an interaction between photons in the same cavity, either actively, as in the polaritonic case, or in an indirect manner, as for the creation of large Kerr nonlinearities. In this section we focus on a complementary regime, in which the tunnelling of photons is used to mediate interactions for atoms in neighbouring cavities. We review proposals to realise effective spin lattice Hamiltonians with individual atoms in micro-cavities that are coupled to each other [27,29,31].

Interacting spin systems have a key role in quantum statistical mechanics, condensed-matter physics, and more recently in quantum information science. On the one hand they have a simple microscopic description, which allows the use of techniques from statistical mechanics in their analysis. On the other, they have a very rich structure [90] and can hence be employed to model and learn about phenomena of real condensed-matter systems. In quantum information science, spin chains can be harnessed to propagate and manipulate quantum information, as well as to implement quantum computation. We first discuss how spin models that conserve the total number of excitations can be generated as limiting cases of Bose-Hubbard type models. Then we review an approach where the full anisotropic Heisenberg Hamiltonian can be engineered for atoms that interact via the exchange of virtual photons. Finally we mention schemes that investigate magnetic properties for photons of different polarisations.

\subsection{Spin models that conserve the total number of excitations}

A certain class of effective spin Hamiltonians can be generated as a limiting case of the effective many-body Hamiltonians discussed in sections 4 and 5 Whenever the engineered on-site interactions are repulsive and strong enough to suppress the occupation of states with more than one excitation almost completely, the states with zero and one excitation can be identified with $|\uparrow\rangle$ and $|\downarrow\rangle$ respectively [22] and the dynamics can effectively be described by the Hamiltonian

$$
H=\sum_{j} B \sigma_{j}^{z}+\sum_{<j, j^{\prime}>} J\left(\sigma_{j}^{x} \sigma_{j^{\prime}}^{x}+\sigma_{j}^{y} \sigma_{j^{\prime}}^{y}\right),
$$

where the second sum runs over nearest neighbours. $\sigma_{j}^{x / y / z}$ are the Pauli matrices at site $j, J$ a coupling coefficient and $B$ a magnetic field. 
The Hamiltonian (35) conserves the number of spins in the state $|\uparrow\rangle$ and can thus be solved efficiently. Much richer spin Hamiltonians can be generated when putting atoms of a $\Lambda$ level structure into the cavities.

\subsection{The anisotropic Heisenberg model}

A particular interesting spin Hamiltonian is the anisotropic Heisenberg or XYZ model, given by

$$
\begin{aligned}
H & =\sum_{j} B \sigma_{j}^{z} \\
& +\sum_{<j, j^{\prime}>} J_{x} \sigma_{j}^{x} \sigma_{j^{\prime}}^{x}+J_{y} \sigma_{j}^{y} \sigma_{j^{\prime}}^{y}+J_{z} \sigma_{j}^{z} \sigma_{j^{\prime}}^{z}
\end{aligned}
$$

This model contains a rich phase diagram and can be used to study quantum magnetism as a limiting case of the fermionic Hubbard model, which is believed to contains the main features of high $T_{c}$ superconductors [90]. From a quantum information perspective, such a model can be used to create cluster states, highly entangled multipartite quantum states which are universal for quantum computation by single-site measurements [91].

The Hamiltonian (36) can be generated in an array of coupled cavities where each cavity contains one atom of a $\Lambda$ level structure, c.f. figure 21 and 22. The two spin polarisations $|\uparrow\rangle$ and $|\downarrow\rangle$ are represented by the two longlived atomic levels of the $\Lambda$ level structures. Together with external lasers, the cavity mode that couples to the atom inside each cavity can induce Raman transitions between these two longlived levels. Due to a large enough detuning between laser and cavity mode, these transitions can only create virtual photons in the cavity mode which mediate an interaction with another atom in a neighbouring cavity. With appropriately chosen detunings, both the excited atomic levels and photon states have vanishing occupation and can be eliminated from the description. As a result, the dynamics is confined to only two states per atom, the longlived levels, and can be described by a spin 1/2 Hamiltonian. The small occupation of photon states and excited atomic levels also strongly suppresses spontaneous emission and cavity decay. As discussed later, this proposal can be realised as soon as the strong coupling regime of cavity QED is achieved.

We note that similar ideas have been recently proposed to realise spin systems of larger spins [31] and with interactions that depend on the direction of the respective lattice edge [29].

In the next subsections we first discuss how effective $\sigma^{x} \sigma^{x}, \sigma^{y} \sigma^{y}$ and $\sigma^{z} \sigma^{z}$ interactions together with an effective magnetic field $B \sigma_{z}$ can be engineered and then explain how these can be combined to generate the full anisotropic Heisenberg model (36).

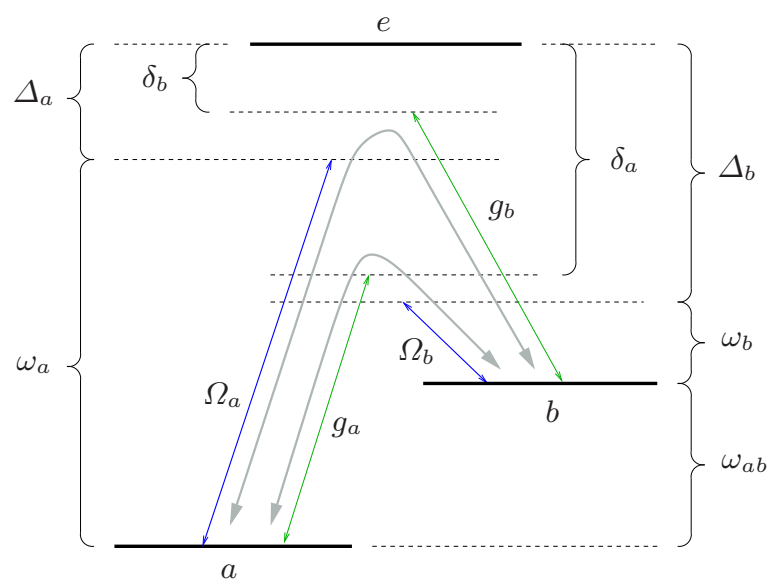

Figure 21 Level structure, driving lasers and relevant couplings to the cavity mode to generate effective $\sigma^{x} \sigma^{x}$ - and $\sigma^{y} \sigma^{y}$ couplings for one atom. The cavity mode couples with strengths $g_{a}$ and $g_{b}$ to transitions $|a\rangle \leftrightarrow|e\rangle$ and $|b\rangle \leftrightarrow|e\rangle$ respectively. One laser with frequency $\omega_{a}$ couples to transition $|a\rangle \leftrightarrow|e\rangle$ with Rabi frequency $\Omega_{a}$ and another laser with frequency $\omega_{b}$ to $|b\rangle \leftrightarrow|e\rangle$ with $\Omega_{b}$. The dominant 2-photon processes are indicated in faint grey arrows. Reprinted with permission from [27].

\subsubsection{XX and YY interactions}

$\mathrm{XX}$ and YY interactions can be generated in an array of coupled cavities with one 3-level atom in each cavity (see figure 21). The two long lived levels, $|a\rangle$ and $|b\rangle$, represent the two spin states. The cavity mode couples to the transitions $|a\rangle \leftrightarrow|e\rangle$ and $|b\rangle \leftrightarrow|e\rangle$, where $|e\rangle$ is the excited state of the atom. Furthermore, two driving lasers couple to the transitions $|a\rangle \leftrightarrow|e\rangle$ respectively $|b\rangle \leftrightarrow|e\rangle$. For the sake of simplicity we consider here a one-dimensional array, but the generalisation to higher dimensions is straight forward. The Hamiltonian of the atoms reads

$$
H_{A}=\sum_{j=1}^{N} \omega_{e}\left|e_{j}\right\rangle\left\langle e_{j}\left|+\omega_{a b}\right| b_{j}\right\rangle\left\langle b_{j}\right|,
$$

where the index $j$ counts the cavities, $\omega_{e}$ is the energy of the excited level and $\omega_{a b}$ the energy of level $|b\rangle$. The energy of level $|a\rangle$ is set to zero. The Hamiltonian that describes the photons in the cavity modes is given by equation. (5). For simplicity of notation we set $J_{C}=2 \omega_{C} \alpha$ and for convenience we assume periodic boundary conditions, where $H_{C}$ can be diagonalised via the Fourier transform

$$
a_{k}=\frac{1}{\sqrt{N}} \sum_{j=1}^{N} \mathrm{e}^{\mathrm{i} k j} a_{j}, \quad k=\frac{2 \pi l}{N}, \quad-\frac{N}{2} \leq l \leq \frac{N}{2}
$$

for $N$ odd to give $H_{C}=\sum_{k} \omega_{k} a_{k}^{\dagger} a_{k}$ with $\omega_{k}=\omega_{C}+$ $2 J_{C} \cos (k)$. Finally the interaction between the atoms and the photons as well as the driving by the lasers are de- 
scribed by

$$
\begin{aligned}
H_{A C}=\sum_{j=1}^{N} & {\left[\left(\frac{\Omega_{a}}{2} \mathrm{e}^{-\mathrm{i} \omega_{a} t}+g_{a} a_{j}\right)\left|e_{j}\right\rangle\left\langle a_{j}\right|\right.} \\
& \left.+\left(\frac{\Omega_{b}}{2} \mathrm{e}^{-\mathrm{i} \omega_{b} t}+g_{b} a_{j}\right)\left|e_{j}\right\rangle\left\langle b_{j}\right|+\text { h.c. }\right] .
\end{aligned}
$$

Here $g_{a}$ and $g_{b}$ are the couplings of the respective transitions to the cavity mode, $\Omega_{a}$ is the Rabi frequency of one laser with frequency $\omega_{a}$ and $\Omega_{b}$ the Rabi frequency of a second laser with frequency $\omega_{b}$ [92]. The complete Hamiltonian is then given by $H=H_{A}+H_{C}+H_{A C}$.

By switching to an interaction picture with respect to $H_{0}=H_{A}+H_{C}-\delta_{1} \sum_{j=1}^{N}\left|b_{j}\right\rangle\left\langle b_{j}\right|$, where $\delta_{1}=\omega_{a b}-$ $\left(\omega_{a}-\omega_{b}\right) / 2$, the excited atom levels $\left|e_{j}\right\rangle$ and the photons can be adiabatically eliminated [93]. To obtain effective spin-spin interactions, terms up to 2 nd order need to be considered while dropping fast oscillating terms. For this approach the detunings $\Delta_{a} \equiv \omega_{e}-\omega_{a}, \Delta_{b} \equiv \omega_{e}-\omega_{b}-$ $\left(\omega_{a b}-\delta_{1}\right), \delta_{a}^{k} \equiv \omega_{e}-\omega_{k}$ and $\delta_{b}^{k} \equiv \omega_{e}-\omega_{k}-\left(\omega_{a b}-\right.$ $\delta_{1}$ ) have to be large compared to the couplings $\Omega_{a}, \Omega_{b}, g_{a}$ and $g_{b}$. Furthermore, the parameters must be such that the dominant Raman transitions between levels $a$ and $b$ are those that involve one laser photon and one cavity photon each (c.f. figure 21). To avoid excitations of real photons via these transitions one requires $\left|\Delta_{a}-\delta_{b}^{k}\right|,\left|\Delta_{b}-\delta_{a}^{k}\right| \gg$ $\left|\frac{\Omega_{a} g_{b}}{2 \Delta_{a}}\right|,\left|\frac{\Omega_{b} g_{a}}{2 \Delta_{b}}\right|$.

Hence whenever the atom emits or absorbs a virtual photon into or from the cavity mode, it does a transition from level $|a\rangle$ to $|b\rangle$ or vice versa. If one atom emits a virtual photon in such a process that is absorbed by a neighbouring atom, which then also does a transition between $|a\rangle$ to $|b\rangle$, an effective spin-spin interaction has happened. Dropping irrelevant constants, the resulting effective Hamiltonian reads

$$
H_{\mathrm{xy}}=\sum_{j=1}^{N} B \sigma_{j}^{z}+\left(J_{1} \sigma_{j}^{+} \sigma_{j+1}^{-}+J_{2} \sigma_{j}^{-} \sigma_{j+1}^{-}+\text {h.c. }\right),
$$

where $\sigma_{j}^{z}=\left|b_{j}\right\rangle\left\langle b_{j}|-| a_{j}\right\rangle\left\langle a_{j}\left|, \sigma_{j}^{+}=\right| b_{j}\right\rangle\left\langle a_{j}\right|$ and $B, J_{1}$ and $J_{2}$ are functions of the various Rabi frequencies and detuning 2 . If $J_{2}^{\star}=J_{2}$, this Hamiltonian reduces to the $\mathrm{XY}$ model,

$$
H_{\mathrm{xy}}=\sum_{j=1}^{N} B \sigma_{j}^{z}+J_{x} \sigma_{j}^{x} \sigma_{j+1}^{x}+J_{y} \sigma_{j}^{y} \sigma_{j+1}^{y},
$$

with $J_{x}=\left(J_{1}+J_{2}\right) / 2$ and $J_{y}=\left(J_{1}-J_{2}\right) / 2$.

\footnotetext{
${ }^{2}$ To second order they are given by $B=\frac{\delta_{1}}{2}-$ $\frac{1}{2}\left[\frac{\left|\Omega_{b}\right|^{2}}{4 \Delta_{b}^{2}}\left(\Delta_{b}-\frac{\left|\Omega_{b}\right|^{2}}{4 \Delta_{b}}-\frac{\left|\Omega_{a}\right|^{2}}{4\left(\Delta_{a}-\Delta_{b}\right)}-\gamma_{b} g_{b}^{2}-\gamma_{1} g_{a}^{2}\right.\right.$ $\left.\left.+\gamma_{1}^{2} \frac{g_{a}^{4}}{\Delta_{b}}\right)-(a \leftrightarrow b)\right], J_{1}=\frac{\gamma_{2}}{4}\left(\frac{\left|\Omega_{a}\right|^{2} g_{b}^{2}}{\Delta_{a}^{2}}+\frac{\left|\Omega_{b}\right|^{2} g_{a}^{2}}{\Delta_{b}^{2}}\right)$ and $J_{2}=\frac{\gamma_{2}}{2} \frac{\Omega_{a}^{\star} \Omega_{b} g_{a} g_{b}}{\Delta_{a} \Delta_{b}}$, where $\gamma_{a, b}=\frac{1}{N} \sum_{k} \frac{1}{\omega_{a, b}-\omega_{k}}, \gamma_{1}=$ $\frac{1}{N} \sum_{k} \frac{1}{\left(\omega_{a}+\omega_{b}\right) / 2-\omega_{k}}$ and $\gamma_{2}=\frac{1}{N} \sum_{k} \frac{\exp (\mathrm{i} k)}{\left(\omega_{a}+\omega_{b}\right) / 2-\omega_{k}}$.
}

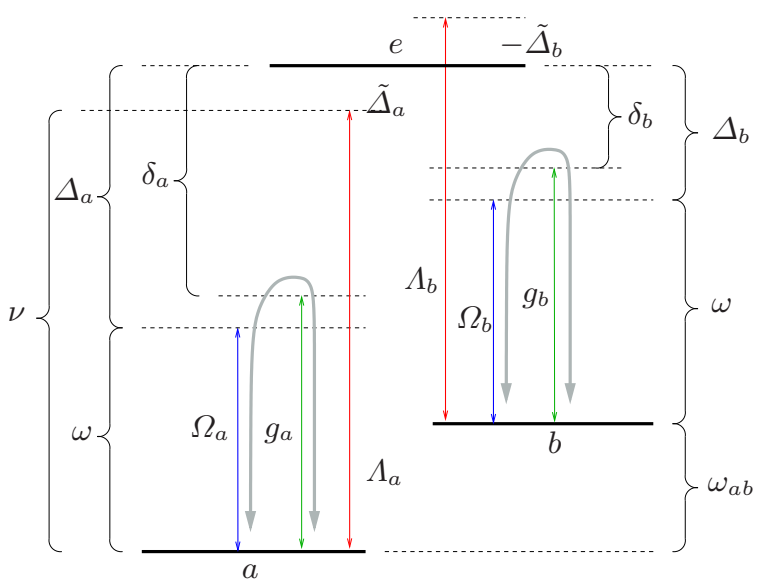

Figure 22 Level structure, driving lasers and relevant couplings to the cavity mode to generate effective $\sigma^{z} \sigma^{z}$-couplings for one atom. The cavity mode couples with strengths $g_{a}$ and $g_{b}$ to transitions $|a\rangle \leftrightarrow|e\rangle$ and $|b\rangle \leftrightarrow|e\rangle$ respectively. Two lasers with frequencies $\omega$ and $\nu$ couple with Rabi frequencies $\Omega_{a}$ respectively $\Lambda_{a}$ to transition $|a\rangle \leftrightarrow|e\rangle$ and $\Omega_{b}$ respectively $\Lambda_{b}$ to $|b\rangle \leftrightarrow|e\rangle$. The dominant 2-photon processes are indicated in faint grey arrows. Reprinted with permission from [27].

For $\Omega_{a}= \pm\left(\Delta_{a} g_{a} / \Delta_{b} g_{b}\right) \Omega_{b}$ with $\Omega_{a}$ and $\Omega_{b}$ real, the interaction is either purely $\sigma^{x} \sigma^{x}(+)$ or purely $\sigma^{y} \sigma^{y}$ $(-)$ and the Hamiltonian (40) becomes the Ising model in a transverse field, whereas the isotropic XY model $\left(J_{x}=\right.$ $J_{y}$, i.e. $J_{2}=0$ ) is obtained for either $\Omega_{a} \rightarrow 0$ or $\Omega_{b} \rightarrow 0$. The effective magnetic field $B$ in turn can, independently of $J_{x}$ and $J_{y}$, be tuned to assume any value between $|B| \gg$ $\left|J_{x}\right|,\left|J_{y}\right|$ and $|B| \ll\left|J_{x}\right|,\left|J_{y}\right|$ by varying $\delta_{1}$. Hence the system can be driven through quantum phase transition by varying the Rabi frequencies and detunings of the driving lasers. Now we proceed to discuss effective ZZ interactions.

\subsubsection{ZZ interactions}

Effective $\sigma^{z} \sigma^{z}$ interactions can be generated with the same atomic level configuration as XX and YY terms, but now only one laser with frequency $\omega$ mediates atom-atom coupling via virtual photons. A second laser with frequency $\nu$ is used to tune the effective magnetic field via a Stark shift. The atoms together with their couplings to cavity mode and lasers are shown in figure 22. Again, we consider the one-dimensional case as an example. The generalisation to higher dimensions is straightforward. The Hamiltonians $H_{A}$ of the atoms and $H_{C}$ of the cavity modes thus have the 
same form as above, whereas $H_{A C}$ now reads

$$
\begin{aligned}
H_{A C} & =\sum_{j=1}^{N}\left[\left(\frac{\Omega_{a}}{2} \mathrm{e}^{-\mathrm{i} \omega t}+\frac{\Lambda_{a}}{2} \mathrm{e}^{-\mathrm{i} \nu t}+g_{a} a_{j}\right)\left|e_{j}\right\rangle\left\langle a_{j}\right|\right. \\
& \left.+\left(\frac{\Omega_{b}}{2} \mathrm{e}^{-\mathrm{i} \omega t}+\frac{\Lambda_{b}}{2} \mathrm{e}^{-\mathrm{i} \nu t}+g_{b} a_{j}\right)\left|e_{j}\right\rangle\left\langle b_{j}\right|+\text { h.c. }\right] .
\end{aligned}
$$

Here, $\Omega_{a}$ and $\Omega_{b}$ are the Rabi frequencies of the driving laser with frequency $\omega$ on transitions $|a\rangle \rightarrow|e\rangle$ and $|b\rangle \rightarrow$ $|e\rangle$, whereas $\Lambda_{a}$ and $\Lambda_{b}$ are the Rabi frequencies of the driving laser with frequency $\nu$ on transitions $|a\rangle \rightarrow|e\rangle$ and $|b\rangle \rightarrow|e\rangle$.

In an interaction picture with respect to $H_{0}=H_{A}+$ $H_{C}$, the excited atom levels $\left|e_{j}\right\rangle$ and the photons can be adiabatically eliminated [93]. Again, the detunings $\Delta_{a} \equiv$ $\omega_{e}-\omega, \Delta_{b} \equiv \omega_{e}-\omega-\omega_{a b}, \tilde{\Delta}_{a} \equiv \omega_{e}-\nu, \tilde{\Delta}_{b} \equiv$ $\omega_{e}-\nu-\omega_{a b}, \delta_{a}^{k} \equiv \omega_{e}-\omega_{k}$ and $\delta_{b}^{k} \equiv \omega_{e}-\omega_{k}-\omega_{a b}$ have to be large compared to the couplings $\Omega_{a}, \Omega_{b}, \Lambda_{a}, \Lambda_{b}, g_{a}$ and $g_{b}$, whereas now Raman transitions between levels $a$ and $b$ should be suppressed. Hence parameters must be such that the dominant 2-photon processes are those that involve one laser photon and one cavity photon each but where the atom does no transition between levels $a$ and $b$ (c.f. figure 22). To avoid excitations of real photons in these processes, one requires $\left|\Delta_{a}-\delta_{a}^{k}\right|,\left|\Delta_{b}-\delta_{b}^{k}\right| \gg\left|\frac{\Omega_{a} g_{a}}{2 \Delta_{a}}\right|,\left|\frac{\Omega_{b} g_{b}}{2 \Delta_{b}}\right|$.

Whenever two atoms exchange a virtual photon in this scheme, both experience a Stark shift that depends on the state of the partner atom. This conditional Stark shift plays the role of an effective $\sigma^{z} \sigma^{z}$-interaction. Dropping irrelevant constants, the effective Hamiltonian reads

$$
H_{\mathrm{zz}}=\sum_{j=1}^{N}\left(\tilde{B} \sigma_{j}^{z}+J_{z} \sigma_{j}^{z} \sigma_{j+1}^{z}\right) .
$$

where $\tilde{B}$ and $J_{z}$ are determined by the detunings and Rabi frequencies of the employed lasers 3 . Here again, the interaction $J_{z}$ and the field $\tilde{B}$ can be tuned independently, either by varying $\Omega_{a}$ and $\Omega_{b}$ for $J_{z}$ or by varying $\Lambda_{a}$ and $\Lambda_{b}$ for $\tilde{B}$. In particular, $\left|\Lambda_{a}\right|^{2}$ and $\left|\Lambda_{b}\right|^{2}$ can for all values of $\Omega_{a}$ and $\Omega_{b}$ be chosen such that either $J_{z} \ll \tilde{B}$ or $J_{z} \gg \tilde{B}$.

Both, $\mathrm{XX}$ and $\mathrm{YY}$ interactions and $\mathrm{ZZ}$ interactions can be combined to engineer the full Hamiltonian (36). This can be done via the Suzuki-Trotter formula.

$$
\begin{aligned}
& 3 \text { To second order they are give by } \tilde{B}=-\frac{1}{2}\left[\frac{\left|\Lambda_{b}\right|^{2}}{16 \Delta_{b}^{2}}\right. \\
& \left(4 \tilde{\Delta}_{b}-\frac{\left|\Lambda_{a}\right|^{2}}{\tilde{\Delta}_{a}-\tilde{\Delta}_{b}}-\frac{\left|\Lambda_{b}\right|^{2}}{\tilde{\Delta}_{b}}-\sum_{j=a, b}\left(\frac{\left|\Omega_{j}\right|^{2}}{\Delta_{j}-\tilde{\Delta}_{b}}+4 \tilde{\gamma}_{j b} g_{j}^{2}\right)\right)+ \\
& \frac{\left|\Omega_{b}\right|^{2}}{16 \Delta_{b}^{2}}\left(4 \Delta_{b}-\frac{\left|\Omega_{a}\right|^{2}}{\Delta_{a}-\Delta_{b}}-\frac{\left|\Omega_{b}\right|^{2}}{\Delta_{b}}-\sum_{j=a, b}\left(\frac{\left|\Lambda_{j}\right|^{2}}{\tilde{\Delta}_{j}-\Delta_{b}}+4 \gamma_{j b} g_{j}^{2}\right)\right. \\
& \left.\left.+4 \gamma_{b b}^{2} \frac{g_{b}^{4}}{\Delta_{b}}\right)-(a \leftrightarrow b)\right] \text { and } J_{z}=\gamma_{2}\left|\frac{\Omega_{b}^{\star} g_{b}}{4 \Delta_{b}}-\frac{\Omega_{a}^{\star} g_{a}}{4 \Delta_{a}}\right|^{2} \\
& \text { with } \gamma_{1}=\frac{1}{N} \sum_{k} \frac{1}{\omega-\omega_{k}}, \quad \gamma_{2}=\frac{1}{N} \sum_{k} \frac{\exp (\mathrm{i} k)}{\omega-\omega_{k}} \text {, } \\
& \left.\gamma_{a a}=\gamma_{b b}=\frac{1}{N} \sum_{k} \frac{1}{\omega-\omega_{k}}, \begin{array}{c}
\gamma_{a b} \\
\gamma_{b a}
\end{array}\right\}=\frac{1}{N} \sum_{k} \frac{1}{\omega \pm \omega_{a b}-\omega_{k}}, \\
& \left.\begin{array}{c}
\tilde{\gamma}_{a b} \\
\tilde{\gamma}_{b a}
\end{array}\right\}=\frac{1}{N} \sum_{k} \frac{1}{\nu \pm \omega_{a b}-\omega_{k}}, \tilde{\gamma}_{a a}=\tilde{\gamma}_{b b}=\frac{1}{N} \sum_{k} \frac{1}{\nu-\omega_{k}} .
\end{aligned}
$$
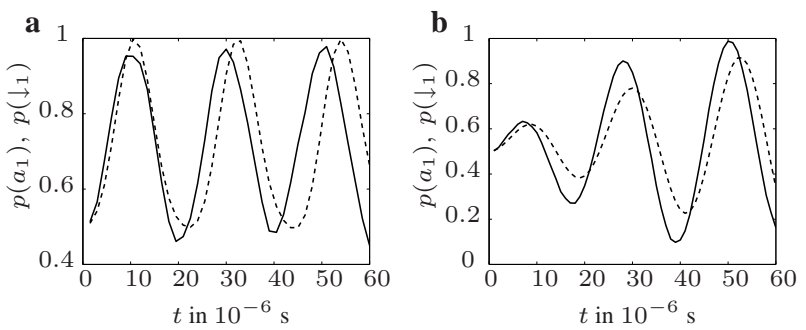

Figure 23 The occupation probability $p\left(a_{1}\right)$ of state $\left|a_{1}\right\rangle$ (solid line) and the probability $p\left(\downarrow_{1}\right)$ of spin 1 to point down (dashed line) for the parameters $\omega_{e}=10^{6} \mathrm{GHz}, \omega_{a b}=30 \mathrm{GHz}, \Delta_{a}=$ $30 \mathrm{GHz}, \Delta_{b}=60 \mathrm{GHz}, \omega_{C}=\omega_{e}-\Delta_{b}+2 \mathrm{GHz}, \tilde{\Delta}_{a}=15 \mathrm{GHz}$, $\Omega_{a}=\Omega_{b}=2 \mathrm{GHz}, \Lambda_{a}=\Lambda_{b}=0.71 \mathrm{GHz}, g_{a}=g_{b}=1 \mathrm{GHz}$, $J_{C}=0.2 \mathrm{GHz}$ and $\delta_{1}=-0.0165 \mathrm{GHz}$ (plot a) respectively $\delta_{1}=$ $-0.0168 \mathrm{GHz}$ (plot b). Both, the occupation of the excited atomic states $\left\langle\mid e_{j}\right\rangle\left\langle e_{j} \mid\right\rangle$ and the photon number $\left\langle a^{\dagger} a\right\rangle$ are always smaller than 0.03. Reprinted with permission from [27].

\subsubsection{The complete effective model}

Making use of the Suzuki-Trotter formula, the two Hamiltonians (40) and 42) can now be combined to one effective Hamiltonian. To this end, the lasers that generate the Hamiltonian (40) are turned on for a short time interval $d t\left(\left\|H_{\mathrm{xy}}\right\| \cdot d t \ll 1\right)$ followed by another time interval $d t$ $\left(|| H_{\mathrm{zz}}|| \cdot d t \ll 1\right)$ with the lasers that generate the Hamiltonian (42) turned on. This sequence is repeated until the total time range to be simulated is covered.

The effective Hamiltonian simulated by this procedure is $H_{\text {spin }}=H_{\mathrm{xy}}+H_{\mathrm{zz}}$, which is precisely the Heisenberg anisotropic model of equation. (36) with $B_{\text {tot }}=B+$ $\tilde{B}$. The time interval $d t$ should thereby be chosen such that $\Omega^{-1}, g^{-1} \ll d t_{1}, d t_{2} \ll J_{x}^{-1}, J_{y}^{-1}, J_{z}^{-1}, B^{-1}$ and $\tilde{B}^{-1}$, so that the Trotter sequence concatenates the effective Hamiltonians $H_{X Y}$ and $H_{Z Z}$. The procedure can be generalised to higher order Trotter formulae or by turning on the sets of lasers for time intervals of different length.

The validity of all above approximations is shown in figure 23. where numerical simulations of the dynamics generated by the full Hamiltonian $H$ are compared it to the dynamics generated by the effective model (40). The present example considers two atoms in two cavities, initially in the state $\frac{1}{\sqrt{2}}\left(\left|a_{1}\right\rangle+\left|b_{1}\right\rangle\right) \otimes\left|a_{2}\right\rangle$, and calculates the occupation probability $p\left(a_{1}\right)$ of the state $\left|a_{1}\right\rangle$ which corresponds to the probability of spin 1 to point down, $p\left(\downarrow_{1}\right)$. Figure 23a shows $p\left(a_{1}\right)$ and $p\left(\downarrow_{1}\right)$ for an effective Hamiltonian (36) with $B_{\text {tot }}=0.135 \mathrm{MHz}, J_{x}=0.065 \mathrm{MHz}$, $J_{y}=0.007 \mathrm{MHz}$ and $J_{z}=0.004 \mathrm{MHz}$ and hence $\left|B_{\text {tot }}\right|>$ $\left|J_{x}\right|$, whereas figure $23 \mathbf{b}$ shows $p\left(a_{1}\right)$ and $p\left(\downarrow_{1}\right)$ for an effective Hamiltonian (36) with the same $J_{x}, J_{y}$ and $J_{z}$ but $B_{\text {tot }}=-0.025 \mathrm{MHz}$ and hence $\left|B_{\text {tot }}\right|<\left|J_{x}\right|[71]$.

Discrepancies between numerical results for the full and the effective model are due to higher order terms for the parameters $B, \tilde{B}, J_{x}, J_{y}$ and $J_{z}$, which lead to relative corrections of up to $10 \%$ in the considered cases. Despite 
this lack of accuracy of the second order approximations, the effective model is indeed a spin-1/2 Hamiltonian as occupations of excited atomic and photon states are negligible.

\subsubsection{Experimental requirements}

For an experimental implementation, the parameters of the effective Hamiltonian, $J_{x}, J_{y}, J_{z}, B$ and $\tilde{B}$ have to be much larger than rates for decay mechanisms via the photons or the excited states $\left|e_{j}\right\rangle$. With the definitions $\Omega=$ $\max \left(\Omega_{a}, \Omega_{b}\right), g=\max \left(g_{a}, g_{b}\right), \Delta=\min \left(\Delta_{a}, \Delta_{b}\right)$, the occupation of the excited levels $\left|e_{j}\right\rangle$ and the photon number $n_{p}$ can be estimated to be $\left\langle\mid e_{j}\right\rangle\left\langle e_{j} \mid\right\rangle \approx|\Omega / 2 \Delta|^{2}$ and $n_{p} \approx\left|(\Omega g / 2 \Delta) \gamma_{1}\right|^{2}$, whereas the couplings $J_{x}, J_{y}$ and $J_{z}$ are approximately $|(\Omega g / 2 \Delta)|^{2} \gamma_{2}$.

Spontaneous emission from the levels $\left|e_{j}\right\rangle$ at a rate $\Gamma_{E}$ and cavity decay of photons at a rate $\Gamma_{C}$ thus lead to effective decay rates

$$
\Gamma_{1}=|\Omega / 2 \Delta|^{2} \Gamma_{E} \text { and } \Gamma_{2}=\left|(\Omega g / 2 \Delta) \gamma_{1}\right|^{2} \Gamma_{C} .
$$

The effective dynamics of Hamiltonian (36) can thus be observed if

$$
\Gamma_{1} \ll|(\Omega g / 2 \Delta)|^{2} \gamma_{2} \text { and } \Gamma_{2} \ll|(\Omega g / 2 \Delta)|^{2} \gamma_{2},
$$

which implies $\Gamma_{E} \ll J_{C} g^{2} / \delta^{2}$ and $\Gamma_{C} \ll J_{C}\left(J_{C}<\right.$ $\delta / 2)$, where, $\delta=\left|\left(\omega_{a}+\omega_{b}\right) / 2-\omega_{C}\right|$ for the XX and YY interactions and $\delta=\left|\omega-\omega_{C}\right|$ for the $\mathrm{ZZ}$ interactions and the approximations $\left|\gamma_{1}\right| \approx \delta^{-1}$ and $\left|\gamma_{2}\right| \approx J_{C} \delta^{-2}$ have been used. Since photons should be more likely to tunnel to the next cavity than decay into free space, $\Gamma_{C} \ll J_{C}$ should hold in most cases. For $\Gamma_{E} \ll J_{C} g^{2} / \delta^{2}$, to hold, cavities with a high ratio $g / \Gamma_{E}$ are favourable. Since $\delta>$ $2 J_{C}$, the two requirements together imply that the cavities should have a high cooperativity factor $\xi$.

\subsection{Magnetic dynamics of polarised light}

In contrast to identifying the internal states of the atoms with spin polarisations, magnetic dynamics can also be studied by considering cavities which support two distinct modes of light with different polarisations, $\uparrow$ and $\downarrow$. In [28] the dynamics of the photon polarisations in such a scheme was analysed. The authors consider cavities that are doped with atoms of a $V$ level structure, where two distinct internal transitions only couple to photons of different polarisations. The interactions of the photons with the atoms are chosen such that double occupancies are suppressed by the blockade effects discussed in section 5 and the dynamics of the photons with creation operators $a_{\uparrow}^{\dagger}$ and $a_{\uparrow}^{\dagger}$ for the two polarisations obeys the effective Hamiltonian [28]

$$
H=\sum_{<j, j^{\prime}>} J\left(S_{j}^{x} S_{j^{\prime}}^{x}+S_{j}^{y} S_{j^{\prime}}^{y}\right)+J^{\prime} S_{j}^{z} S_{j^{\prime}}^{z},
$$
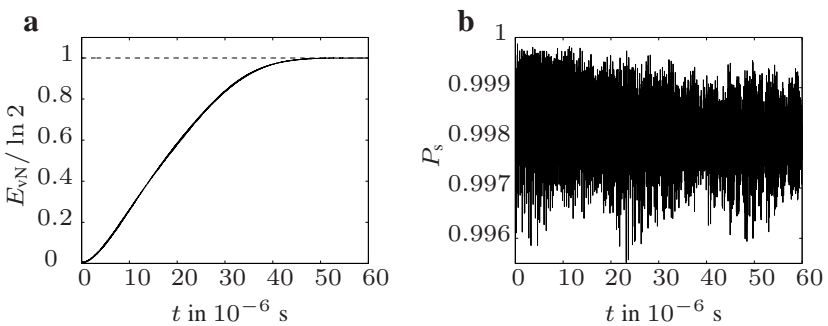

Figure 24 a: The von Neumann entropy $E_{\mathrm{vN}}$ of the reduced density matrix of 1 effective spin in multiples of $\ln 2$ and $\mathbf{b}$ : the purity of the reduced state of the effective spin model for 3 cavities where $J_{z}=0.021 \mathrm{MHz}$. The plots assume that no spontaneous emission took place. For a spontaneous emission rate of $0.1 \mathrm{MHz}$ $(g=1 \mathrm{GHz})$, the probability for a decay event in the total time range is $1.5 \%$. Hence, cluster state generation fails with probability $0.005 \times n$ for $n \ll 1 / 0.005=200$ cavities, irrespectively of the lattice dimension. Reprinted with permission from [27].

where $S_{j}^{z}=\frac{1}{2}\left(a_{j \uparrow}^{\dagger} a_{j \uparrow}-a_{j \downarrow}^{\dagger} a_{j \downarrow}\right), S_{j}^{x}=\frac{1}{2}\left(a_{j \uparrow}^{\dagger} a_{j \downarrow}+a_{j \downarrow}^{\dagger} a_{j \uparrow}\right)$, $S_{j}^{y}=\frac{1}{2 \mathrm{i}}\left(a_{j \uparrow}^{\dagger} a_{j \downarrow}-a_{j \downarrow}^{\dagger} a_{j \uparrow}\right)$ and the indices $j$ and $j^{\prime}$ label the cavities.

\subsection{Cluster state generation}

Effective spin models in arrays of coupled cavities can be used for the generation of cluster states [91], which form, together with the local addressability, a platform for oneway quantum computation 94,95 . The experimental verification that the desired entanglement has indeed been created is thereby a non-trivial task in its own right [96].

One way to generate cluster states is via the effective Hamiltonian (42] [27]. A similar rout has been presented in [30]. To this end, all atoms are initialised in the states $\left(\left|a_{j}\right\rangle+\left|b_{j}\right\rangle\right) / \sqrt{2}$, which can be done via a STIRAP process [76], and then evolved under the Hamiltonian (42) for $t=$ $\pi / 4 J_{z}$.

Figure 24 shows the von Neumann entropy of the reduced density matrix of one effective spin $E_{\mathrm{vN}}$ and the purity of the reduced density matrix of the effective spin chain $P_{\mathrm{s}}$ for a full three cavity model. Since $E_{\mathrm{vN}} \approx \log _{2} 2$ for $t \approx 50 \mu$ s while the state of the effective spin model remains highly pure $\left(P_{\mathrm{s}}=\operatorname{tr}\left[\rho^{2}\right]>0.995\right)$ the degree of entanglement will be very close to maximal. Thus the levels $\left|a_{j}\right\rangle$ and $\left|b_{j}\right\rangle$ have been driven into a state which is, up to local unitary rotations, very close to a three-qubit cluster state.

A second way to generate cluster states is to implement controlled phase gates between the qubits in different cavities [52]. First all qubits are initialised in the state $|+\rangle=(|1\rangle+|0\rangle) / \sqrt{2}$, where $|1\rangle$ and $|0\rangle$ refer to states with one or zero excitations in a cavity. The phase gates are then implemented by isolating chains of three qubits via a Stark shift that turns surrounding qubits off resonance. The isolated three-qubit chains evolve for a time $t=\pi /(2 \sqrt{2} J)$ 


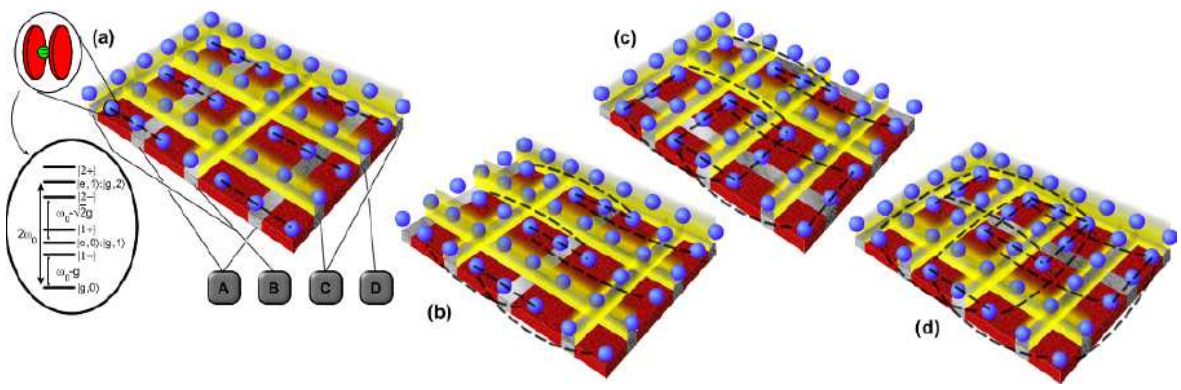

Figure 25 A 2d cavity array. Consecutive application of gates $\mathrm{A}, \mathrm{B}$, $\mathrm{C}$ and $\mathrm{D}$, depicted in parts (a), (b), (c) and (d), each isolate chains of 3 qubits, realising controlledphases and SWAPs (swapping quantum/classical gates) between the qubits at either end of the chain (the dashed lines indicate where the controlled-phase gates have been applied). Reprinted with permission from [52].
( $J$ is the photon tunnelling rate) and the central qubit is subsequently measured. Local unitaries on the remaining two qubits that depend on the outcome of this measurement complete the controlled phase gate. Figure 25 shows the entire sequence of gates.

Further ways to create entangled states include measurement based schemes. One version uses two atoms in two cavities that interact via the exchange of photons [55]. Both atoms can emit photons of two polarisations (left circular and right-circular), depending on which excited state they are in. Hence the two atoms are prepared such that they each emit a single photon, one with left-circular and one with right-circular polarisation. After a time $t=\pi / 4 J$ ( $J$ being the photon tunnelling rate), when photons have tunnelled between the cavities with probability $1 / 2$, the two atoms are employed to perform a non-demolition measurement to determine the number of photons in each cavity. Provided each cavity contains one photon the state is collapsed to a two photon polarisation entangled state thus providing a heralded source of entangled photons.

Apart from quantum information motivated applications coupled cavity arrays with local addressability can also be used to study novel physical setups. Among those are interface between regions in different condensed matter phases, see section 9

\section{Promising Platforms for Realisations}

In this section, we present some cavity QED setups which have favourable features for the realisation of the effective many-body models described in this review. These platforms are thus promising candidates for experimental demonstrations of the theory presented here. The selection we present here obviously reflects our personal view. It is of course possible that devices which we do not explicitly mention here may in the end provide the first realisation of polaritonic many-body systems as discussed in previous sections.

A major part of all cavity QED experiments have been performed with Fabry-Pérot cavities formed by rather massive mirrors. With such mirrors, coupling two cavities such that photons can tunnel between them seems quite difficult. On the other hand several new cavity QED structures with small volume micro-cavities have emerged in recent years. These are routinely produced in large arrays and by construction can allow for efficient photon tunnelling between neighbouring cavities. In addition, the strong coupling regime with cooperativity factors much larger than unity has been achieved in several of these devices. These two features together make such micro-cavity arrays very promising candidates for the generation of the effective many-body models discussed in this review. In the following we discuss some platforms in a bit more detail.

\subsection{Photonic band gap cavities}

One promising device consists of quantum dots coupled to photonic band gap defect nanocavities. Photonic crystals are structures with periodic dielectric properties which affect the motion of photons, in a similar manner as the periodicity of a semiconductor affects the motion of electrons [97]. Such structures can exhibit band gaps in frequency space in which no photon of particular frequencies can propagate in the material (for an introductory review see [98]).

A nanocavity can be created in a photonic-band-gap material by producing a localised defect in the structure of the crystal, in such a way that light of a particular frequency cannot propagate outside the defect area. Large arrays of such nanocavities have been produced [99]. Photon hopping between neighbouring cavities has been observed in the microwave and optical domains [100, 101], where its description in the form as given in equation (5) was found to be accurate. Quantum dots, semiconductors whose excitations are confined in all three directions, behave in many respects like atoms and can be effectively addressed by lasers [102]. Quantum dots can be created inside photonic crystal nanocavities [103] and made to interact with the cavity mode to form a standard cavity QED system. The strong coupling regime with cooperativity factors up to $\xi \approx 10$ has already been achieved in such systems [61]. Cooperativity factors of $\xi \sim 10^{6}$ are predicted to be realisable [81]. Due to the extremely small volume of the nanocavity, the Jaynes-Cummings coupling coefficient can be extremely large [60,104], while spontaneous emission rates are very low. Figure 26 shows a quantum dot in a 


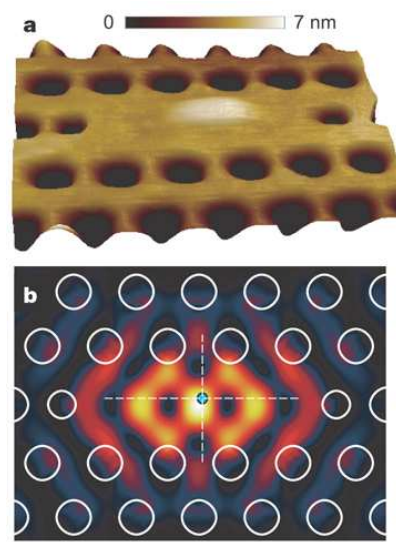

Figure 26 A quantum dot in a photonic band gap cavity. a, AFM topography of a photonic crystal nanocavity aligned to a hill of material on the surface arising from a quantum dot buried $63 \mathrm{~nm}$ below. b, Electric field intensity of the photonic crystal cavity mode showing that the location of the buried $\mathrm{QD}$, indicated by the teal dot, overlaps the field maximum. Reprinted with permission from [61].

photonic band gap cavity. For the applications discussed in this review current cavity decay rates of photonic band gap cavities remain a limiting factor. This is mainly due to the fact that most structures employ two dimensional photonic crystals as placing a quantum dot into a three dimensional structure is difficult. The difficulties to control the properties of individual quantum dots and to place them at desired locations could even pose a more significant problem. Current experiments pick a quantum dot from an ensemble and then fabricate a photonic crystal tailored to this dot around it. Such an approach can obviously not be used for producing a whole array of sufficient quality.

One might think about trapping atoms in photonic band gap cavities instead of letting them interact with quantum dots. Since the frequencies of currently available photonic band gaps do not fit to atomic transition frequencies, such a strategy would require the development of new photonic band gap materials.

\subsection{Silicon structures: micro discs and micro toroids}

A second class of micro-cavities that are routinely produced in large arrays and which have very high Q-factors are silicon structures of either a disc [59] or a toroidal [57] shape, see figure 27. These cavities are closed silicon discs or toroids that typically are carried by a thin pillar in their centre. They are routinely produced in large arrays [105]. The light is trapped in whispering gallery modes that are localised close to the outer surface of the structure and have a small mode volume [106]. Very efficient and highly tunable coupling of photons into and from those cavities is possible via tapered optical fibres that are placed close to their surface [107]. Here the evanescent field of both cavity and fibre overlap and photons can hop between both. The photon hopping rate can thereby be controlled very accurately via the separation between cavity and fibre.

These cavities can interact with atoms in a strong coupling regime with cooperativity factors $\xi \sim 50[58]$ if the

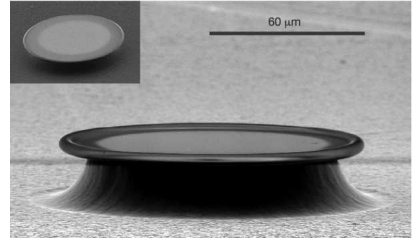

Figure 27 SEM picture of a toroidal microresonator with an intrinsic cavity Q of $10^{8}$. Reprinted with permission from [57].

atoms are placed close to the cavity surface and interact with the evanescent field. This has been seen in an experiment where atoms fell by the cavity surface [58] and a photon blockade effect generated by one atom has been demonstrated [108]. Theoretical predictions [81] show that very large cooperativity factors of $\xi \sim 10^{6}$ are expected to be achieved in such systems.

For realising effective Hamiltonians such as (7), 30 or 36), some aspects still remain challenging for micro disc and micro toroidal cavities. One aspect is that atoms need to be trapped for a sufficiently long time close enough to the cavity surface to maintain a sufficient strong coupling regime. A second aspect is that all cavities of an array need to be tuned into resonance with each other with enough accuracy. This can be achieved by varying their temperature and thus shrinking or expanding them, but is still a technical challenge. Finally there can be modifications of the photon hopping as compared to the form assumed in equation (5). A challenge here would be to prevent that significant photon population is localised in the fibre instead of in the cavities themselves. Nonetheless, even if the fibre contains appreciable excitations, interesting many-body physics is expected to happen as the fibre would merely play the role of an additional lattice site with different local terms than the other sites.

Figure 28 shows an array of 10 micro disc cavities that are all coupled by one tapered optical fibre and mounted on an atom chip. The purpose of the atom chip is to trap atoms close to the cavity surfaces and, in that way, maintain a strong coupling regime for substantial times.

\subsection{Fibre based cavities}

Strong coupling experiments with many atoms in one cavity and at the same time large cooperativity factors for each individual atom have been performed with fibre FabryPérot cavities [64]. These cavities are formed by two tips of optical fibres that are facing each other and are mounted on an atom chip to trap an manipulate the atoms that interact with the cavity modes. Current chips contain two such cavities as shown in figure 29 and cooperativity factors as high as $\xi=145$ have been achieved [64].

Photons are coupled into and from the cavity directly via the optical fibres. Hence as for fibre coupled micro discs or toroids this may lead to modifications of the form of the photon hopping as compared to equation (5). On the other hand, the atoms are here trapped on the atom chip 


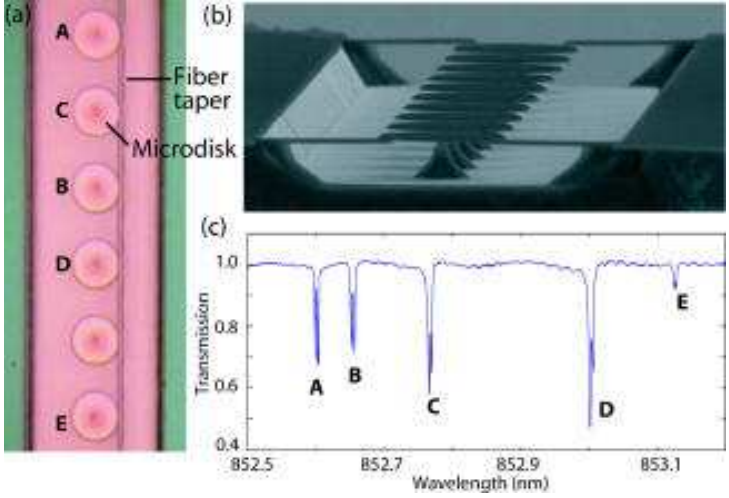

Figure 28 (a) Top-view optical image of a fibre taper aligned with and array of ten micro-disks (the remaining four micro-disks are out of the field of view of the image). (b) SEM image of the array of ten micro-disks. (c) Fibre taper transmission vs wavelength when the taper is aligned with the micro-disk array in (a). The letters match specific micro-disks in (a) with the corresponding resonances in (c). Reprinted with permission from [59].

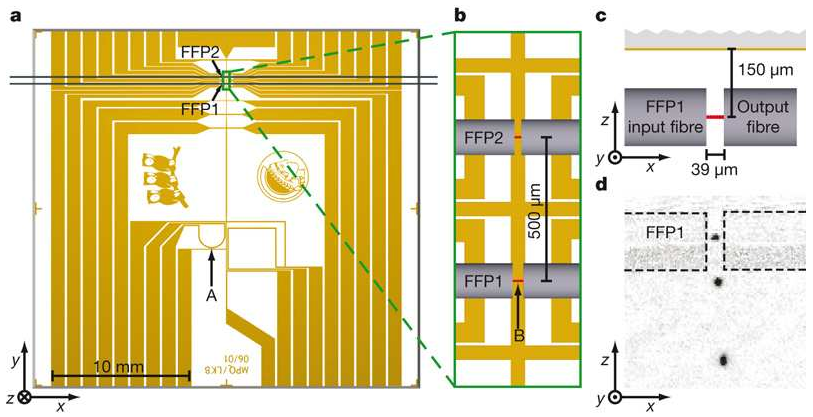

Figure 29 a, Layout of an atom chip that contains two fibre FabryPérot cavities. b, Close-up view of the two fibre FabryPérot (FFP) optical cavities that are mounted on the chip. Cavity modes are drawn to scale in red. The BEC is produced in a magnetic trap and positioned in the FFP1 mode ('B'). c, Geometry of the FFP1 cavity. d, Overlay of three CCD time-of-flight (TOF) absorption images, showing the anisotropic expansion of a BEC having interacted for $50 \mathrm{~ms}$ with the cavity field. The optical fibres are outlined for clarity. Reprinted with permission from [64].

and can thus be held in a location of strong interaction with the cavity modes for a long time.

\subsection{On-chip Fabry-Pérot cavities}

Micro-fabricated arrays of Fabry-Pérot type cavities with large cooperativity factors have been presented in [109]. Each plano-concave resonator consists of an etched spherical mirror and a coated single-mode optical fibre, allowing open access to the intra-cavity field mode. Here, large

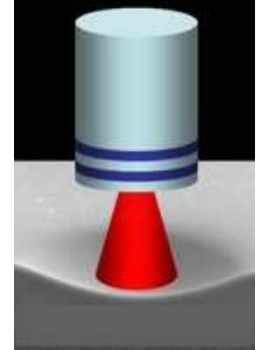

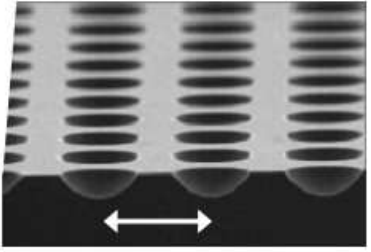

$90 \mu \mathrm{m}$
Figure 30 Left: Single on-chip Fabry-Pérot cavity formed by an etched micro-mirror and a tip of an optical fibre. Right: Two dimensional array of cavity micro-mirrors. Reprinted with permission from [110].

regular arrays of spherical mirrors can be created with controllable sizes and spacings. These cavities which have a very small mode volume are illustrated in figure 30. The extremely small mode volume leads to a large single-photon Rabi frequency, and light is conveniently coupled into and out of the cavities via the fibres themselves. These cavities to date achieve a finesse $F \sim 5000$, corresponding to Purcell factors (or cooperativities) $\xi \sim 40$.

As with the other cavities that involve optical fibres modifications of the photon hopping as compared to equation (5) are expected here as well. Current strong coupling experiments have also been only performed with falling atoms 63.

\subsection{Superconducting stripline resonators}

A promising experimental platform that operates in the microwave regime are cavity QED system formed by a Cooper-pair box coupled to a superconducting transmission line resonator. A Cooper-pair box is formed by two superconducting islands separated by a Josephson junction. Although it is a macroscopic device, it behaves in many ways like an atom as the relevant structure can be described by a two level quantum system. A superconducting transmission line cavity, on the other hand, is a quasi-onedimensional coplanar waveguide resonator, formed on a chip with a resonance frequency in the microwave range. The strong-coupling regime in such a system has been achieved [62] and recently two Cooper-pair boxes have been strongly coupled to the cavity mode [111,112]. Very large cooperativity factors have been achieved in this set-up [62,112] and the nonlinearity of the Jaynes-Cummings model has been measured [113]. Although coupling between different cavities has not been realised, up to ten Cooper-pair boxes interacting in the strong coupling regime with the resonant cavity mode has been predicted to be achievable using the architecture of Ref. [112]. 


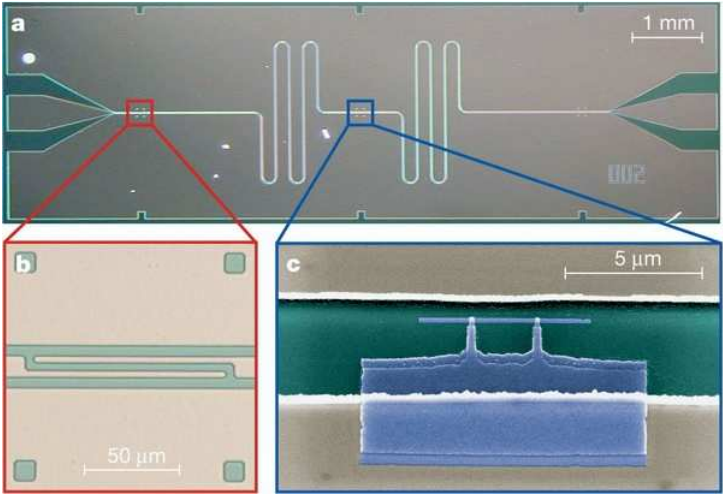

Figure 31 a, A superconducting niobium coplanar waveguide resonator of $24 \mathrm{~mm}$ length. It is coupled by a capacitor at each end of the resonator (see b) to an input and output feed line. $\mathbf{b}$, The capacitive coupling to the input and output lines. c, False colour electron micrograph of a Cooper pair box (blue) fabricated onto the silicon substrate (green) into the gap between the centre conductor (top) and the ground plane (bottom) of a resonator (beige) using electron beam lithography and double angle evaporation of aluminium. Reprinted with permission from [62].

\section{Applications}

Structures of coupled arrays of micro-cavities have several interesting potential applications. Particularly useful in this respect is the individual addressability of single cavities with optical lasers. The feasibility of single site addressing is based on the size of the cavities which can easily be larger than an optical wavelength. Furthermore the inter-cavity coupling could be realised by optical fibres which would move the cavities even further apart.

Among the potential applications are the implementation of tools for quantum information processing. These include the generation of entangled states such as cluster states [27,52,54,55] (see section 7.4), the controlled propagation of polaritonic excitations in cavity arrays [53, 35. 36, 37, 50], quantum gates [51] and the generation of single photons in parallel [56].

Perhaps most importantly, the local controllability offered by coupled cavity arrays can also give rise to novel physical effects and we will mention here one example of these. Local manipulations of an effective many body system could be applied to engineer a boundary between a Mott insulator and a superfluid region of a Bose-Hubbard model at unit filling. Initially both regions are decoupled and cooled to their respective ground states. This situation can for example be prepared in a cavity array that is operated in the way as discussed in section 4 and thus hosts a polaritonic Bose-Hubbard Hamiltonian (7). After switching on a small tunnelling rate between both regions, all particles of the Mott region migrate to the superfluid area. This behaviour is shown for a one dimensional chain in figure 32, where the particle densities for all lattice sites

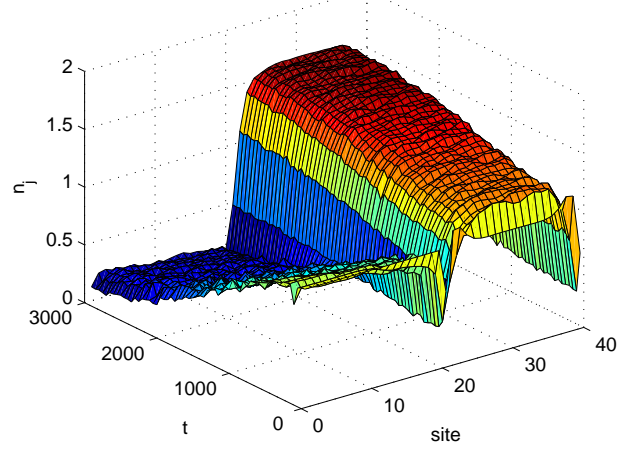

Figure 32 The particle density across the chain as function of time. Sites 1 to 20 start in a Mott insulator regime and the rest in a superfluid regime. $U=1.0, J=0.1, \mu=0, \tilde{U}=0.2, \tilde{\mu}=0$, $\tilde{J}=1.0$ and $J_{I}=0.1$. Reprinted with permission from [33].

are shown as a function of time. The migration takes place whenever the difference between the chemical potentials of both regions is less than the maximal energy of any eigenmode of the superfluid [33].

\section{Summary}

In this review, we have tried to give an overview of the current status of approaches to engineer effective many body dynamics in arrays of cavities that are coupled via photon tunnelling. The schemes that have been developed so far can be divided into two main categories, effective BoseHubbard type models and effective spin models.

In Bose-Hubbard type models, quantum phase transitions between superfluid phases of polaritons or photons (coherent light) and Mott insulators can be studied. Here, the experimentally challenging and hence physically new and interesting part is to create a Mott insulator in which polaritons or photons are by their effective mutual repulsion forced to stay in one lattice site (cavity) and not able to move. This situation would in correspond to a crystal formed by light or "frozen light".

A key issue on the experimental side is to generate a Kerr nonlinearity or effective interaction between polaritons/photons that is significantly larger than the decay mechanisms present in the cavity array.

Existing approaches for effective spin models mainly employ two metastable levels of an atom trapped in each cavity to represent the two spin polarisations $\uparrow$ and $\downarrow$. Raman transitions mediated by external driving lasers that give rise to interactions between neighbouring cavities via the exchange of virtual photons are then used to generate effective spin-spin interactions.

As for Bose Hubbard type models a main experimental challenge is to make atom-photon interactions signif- 
icantly stronger than the decay mechanisms in the cavity array.

Effective many body system in a coupled cavity arrays are of great interest from various points of view. First of all they open up a way to study condensed matter physics with light and hence to engineer new quantum states of light. Furthermore they offer a great versatility for applications as quantum simulators due to the possibilities to manipulate them via external driving lasers. Most importantly they would, by construction, allow for control, manipulations and measurements of individual lattice sites. The situation is somehow complementary to cold atoms in optical lattices, where very large lattices can be created and experiments provide good measurement access to global quantities such as the phase coherence of the superfluid.

A polaritonic many body system in a cavity array on the other hand will for the foreseeable future only comprise much smaller lattices, but provide good access for the measurement of local quantities. In contrast to optical lattices it would thus allow to directly verify the integer occupation number of each lattice site in the Mott insulator regime.

The local addressability of coupled cavity arrays gives rise to a number of novel application. These contain quantum information processing application but also novel physical situations that give rise to features such as particle migration.

Acknowledgements This work is part of the QIP-IRC supported by EPSRC (GR/S82176/0), the Integrated Project Qubit Applications (QAP) supported by the IST directorate as Contract Number 015848', the EU STREP project HIP and was supported by the EPSRC grant EP/E058256/1, the Alexander von Humboldt Foundation, the Conselho Nacional de Desenvolvimento Científico e Tecnológico (CNPq), the Royal Society and the DFG Emmy Noether grant HA 5593/1-1.

\section{References}

[1] H.J. Metcalf and P. van der Straten, Laser Cooling and Trapping. Springer, New York, (1999).

[2] A.J. Leggett, Bose-Einstein condensation in the alkali gases: Some fundamental concepts, Rev. Mod. Phys. 73, 307 (2001).

[3] D. Leibfried, R. Blatt, C. Monroe, D. Wineland, Quantum dynamics of single trapped ions, Rev. Mod. Phys., 75, 281 (2003).

[4] J.M. Raimond, M. Brune and S. Haroche, Manipulating quantum entanglement with atoms and photons in a cavity, Rev. Mod. Phys. 73, 565 (2001).

[5] A. Zeilinger, Experiment and the foundations of quantum physics, Rev. Mod. Phys. 71, S288 (1999).

[6] F. De Martini and C. Monroe (editors), Experimental Quantum Computation and Information, IOS Press, Amsterdam, (2002).

[7] R.P. Feynman, Simulating physics with computers, Inter. J. Theor. Phys. 21, 467 (1982).
[8] I. Bloch, J. Dalibard and W. Zwerger, Many-Body Physics with Ultracold Gases, Rev. Mod. Phys. 80, 885 (2008)

[9] D. Jaksch, C. Bruder, J.I. Cirac, C.W. Gardiner and P. Zoller, Cold Bosonic Atoms in Optical Lattices, Phys. Rev. Lett. 81, 3108 (1998)

[10] M. Greiner, O. Mandel, T. Esslinger, T.W. Hänsch and I. Bloch, Quantum phase transition from a superfluid to a Mott insulator in a gas of ultracold atoms, Nature 415, 39 (2002).

[11] B. Paredes, A. Widera, V. Murg, O. Mandel, S. Flling, I. Cirac, G.V. Shlyapnikov, T.W. Hänsch and I. Bloch, TonksGirardeau gas of ultracold atoms in an optical lattice, Nature 429, 277 (2004).

[12] M. Lewenstein, A. Sanpera, V. Ahufinger, B. Damski, A. Sen, U. Sen, Ultracold atomic gases in optical lattices: mimicking condensed matter physics and beyond, Advances in Physics 56, 243 (2007).

[13] Y. Makhlin, G. Schön, and A. Shnirman, Quantum-state engineering with Josephson-junction devices, Rev. Mod. Phys. 73, 357 (2001).

[14] H.S. van der Zant, F.C. Fritschy, W.J. Elion, L.J. Geerligs and J.E. Mooij, Field-induced superconductor-to-insulator transitions in Josephson-junction arrays, Phys. Rev. Lett. 69, 2971 (1992).

[15] A. van Oudenaarden and J.E. Mooij, One-Dimensional Mott Insulator Formed by Quantum Vortices in Josephson Junction Arrays, Phys. Rev. Lett. 76, 4947 (1996).

[16] F. Mintert and C. Wunderlich, Ion-Trap Quantum Logic Using Long-Wavelength Radiation, Phys. Rev. Lett. 87, 257904 (2001).

[17] D. Porras and J.I. Cirac, Effective Quantum Spin Systems with Trapped Ions, Phys. Rev. Lett. 92, 207901 (2004).

[18] D. Porras and J.I. Cirac, Bose-Einstein Condensation and Strong-Correlation Behavior of Phonons in Ion Traps, Phys. Rev. Lett. 93, 263602 (2004).

[19] A. Retzker, R. Thompson, D. Segal and M.B. Plenio, Double wells, scalar fields and quantum phase transitions in ions traps, arXiv:0801.0623

[20] A. Friedenauer, H. Schmitz, J.T. Glückert, D. Porras and T. Schätz, Simulating the Quantum Magnet, arXiv:0802.4072

[21] M.J. Hartmann, F.G.S.L. Brandão and M.B. Plenio, Strongly interacting polaritons in coupled arrays of cavities, Nature Phys. 2, 849 (2006).

[22] D.G. Angelakis, M.F. Santos and S. Bose, Photon-blockadeinduced Mott transitions and XY spin models in coupled cavity arrays, Phys. Rev. A 76, R031805 (2007).

[23] A.D. Greentree, C. Tahan, J.H. Cole and L.C.L. Hollenberg, Quantum phase transitions of light, Nature Phys. 2, 856 (2006).

[24] F. Illuminati, Quantum optics: Light does matter, Nature Phys. 2, 803 (2006).

[25] M.J Hartmann and M.B Plenio, Strong Photon Nonlinearities and Photonic Mott Insulators, Phys. Rev. Lett. 99, 103601 (2007).

[26] F.G.S.L. Brandão, M.J. Hartmann and M.B. Plenio, Lightshift-induced photonic nonlinearities, New J. Phys. 10, 043010 (2008).

[27] M.J. Hartmann, F.G.S.L. Brandão and M.B. Plenio, Effective Spin Systems in Coupled Microcavities, Phys. Rev. Lett. 99, 160501 (2007).

[28] A.-C. Ji, X.C. Xie and W.M. Liu, Quantum Magnetic Dynamics of Polarized Light in Arrays of Microcavities, Phys. Rev. Lett. 99, 183602 (2007). 
[29] A. Kay and D.G. Angelakis, Reproducing spin lattice models in strongly coupled atom-cavity systems, arXiv:0802.0488

[30] P. Li, Q. Gong and G. Guo, Effective generation of Ising interaction and cluster states in coupled micro-cavities, arXiv:0712.3864

[31] J. Cho, D.G. Angelakis and S. Bose, Simulation of high-spin Heisenberg chains in coupled cavities, arXiv:0802.3365

[32] J. Cho, D.G. Angelakis and S. Bose, Fractional Quantum Hall State in Coupled Cavities, arXiv:0807.1802

[33] M.J. Hartmann and M.B. Plenio, Migration of Bosonic Particles across a Mott Insulator to a Superfluid Phase Interface, Phys. Rev. Lett. 100, 070602 (2008).

[34] C.D. Ogden, E.K. Irish and M.S. Kim, Dynamics in a coupled-cavity array, arXiv:0804.2882

[35] L. Zhou, J. Lu and C.P. Sun, Coherent control of photon transmission: Slowing light in a coupled resonator waveguide doped with $\Lambda$ atoms, Phys. Rev. A 76, 012313 (2007).

[36] F.M. Hu, L. Zhou, T. Shi and C.P. Sun, Coupled cavity QED for coherent control of photon transmission: Green-function approach for hybrid systems with two-level doping Phys. Rev. A 76, 013819 (2007).

[37] L. Zhou, Y.B. Gao, Z. Song and C.P. Sun, Coherent output of photons from coupled superconducting transmission line resonators controlled by charge qubits, Phys. Rev. A 77, 013831 (2008).

[38] M.J. Hartmann, F.G.S.L. Brandão and M.B. Plenio, A polaritonic two-component BoseHubbard model, New J. Phys. 10, 033011 (2008).

[39] D. Rossini and R. Fazio, Mott-Insulating and Glassy Phases of Polaritons in 1D Arrays of Coupled Cavities, Phys. Rev. Lett. 99, 186401 (2007).

[40] M. Aichhorn, M. Hohenadler, C. Tahan and P. B. Littlewood, Quantum Fluctuations, Temperature, and Detuning Effects in Solid-Light Systems, Phys. Rev. Lett. 100, 216401 (2008).

[41] E.K. Irish, C.D. Ogden, and M.S. Kim, Polaritonic characteristics of insulator and superfluid states in a coupled-cavity array, Phys. Rev. A 77, 033801 (2008).

[42] M.I. Makin, J.H. Cole, C. Tahan, L.C.L. Hollenberg and A.D. Greentree, Quantum phase transitions in photonic cavities with two-level systems, Phys. Rev. A 77, 053819 (2008).

[43] J. Zhao, A.W. Sandvik and K. Ueda, Insulator to superfluid transition in coupled photonic cavities in two dimensions, arXiv:0806.3603

[44] N. Na, S. Utsunomiya, L. Tian and Y. Yamamoto, Strongly correlated polaritons in a two-dimensional array of photonic crystal micro-cavities, Phys. Rev. A 77, 031803(R) (2008).

[45] D.E. Chang, V. Gritsev, G. Morigi, V. Vuletic, M.D. Lukin and E.A. Demler, Crystallization of strongly interacting photons in a nonlinear optical fiber arXiv:0712.1817

[46] M. Paternostro, G.S. Agarwal and M.S. Kim, Solitons in interacting Dicke models of coupled cavities with two-level systems, arXiv:0707.0846

[47] S.-C. Lei and R.-K. Lee, Quantum phase transitions of light in the Dicke-Bose-Hubbard model, Phys. Rev. A 77, 033827 (2008).

[48] Y.-F. Xiao, J. Gao, X.-B. Zou, J.F. McMillan, X. Yang, Y.L. Chen, Z.-F. Han, G.-C. Guo and C.W. Wong, Coupled quantum electrodynamics in photonic crystal nanocavities, arXiv.org:0707.2632
[49] D. Rossini, R. Fazio and G. Santoro, Photon and polariton fluctuations in arrays of QED-cavities, Europhys. Lett. in press, arXiv:0806.0942

[50] M.X. Huo, Y. Li, Z. Song and C.P. Sun, Atomic entanglement versus visibility of photon interference for quantum criticality of a hybrid system, Phys. Rev. A 77, 022103 (2008).

[51] D.G. Angelakis, M.F. Santos, V. Yannopapas and A. Ekert, A proposal for the implementation of quantum gates with photonic-crystal coupled cavity waveguides, Phys. Lett. A. 362, 377 (2007).

[52] D.G. Angelakis and A. Kay, Weaving light-matter qubits into a one way quantum computer, New J. Phys. 10, 023012 (2008).

[53] S. Bose, D.G. Angelakis and D. Burgarth, Transfer of a Polaritonic Qubit through a Coupled Cavity Array, Journ. of Mod. Opt. 54, 2307 (2007).

[54] D.G. Angelakis, Mancini and S. Bose, Steady state entanglement between hybrid light-matter qubits, arXiv.org:0711.1830

[55] J. Cho, D.G. Angelakis and S. Bose, Heralded generation of two-photon polarization entanglement with coupled cavities, Phys. Rev. A 78, 022323 (2008).

[56] N. Na and Y. Yamamoto, Generation of indistinguishable single photons and polarization-entangled photon-pairs via polaritonic superfluid to Mott insulator quantum phase transition, arXiv:0804.1829

[57] D.K. Armani, T.J. Kippenberg, S.M. Spillane and K.J. Vahala, Ultra-high-Q toroid micro-cavity on a chip, Nature 421, 925 (2003).

[58] T. Aoki, B. Dayan, E. Wilcut, W.P.Bowen, A.S. Parkins, H.J. Kimble, T.J. Kippenberg and K.J. Vahala, Observation of strong coupling between one atom and a monolithic microresonator, Nature 443, 825 (2006).

[59] P.E. Barclay, K. Srinivasan, O. Painter, B. Lev and H. Mabuchi, Integration of fiber-coupled high-Q $\mathrm{SiN}_{x}$ microdisks with atom chips Appl. Phys. Lett. 89, 131108 (2006).

[60] Y. Akahane, T. Asano, B.-S.Song and S. Noda High-Q photonic nanocavity in a two-dimensional photonic crystal, Nature 425, 944 (2003).

[61] K. Hennessy, A. Badolato, M. Winger, D. Gerace, M. Atatüre, S. Gulde, S. Fält, E.L. Hu and A. Imamoğlu, Quantum nature of a strongly coupled single quantum dot - cavity system, Nature 445, 896 (2007).

[62] A. Wallraff, D.I. Schuster, A. Blais, L. Frunzio, R.S. Huang, J. Majer, S. Kumar, S.M. Girvin and R.J. Schoelkopf, Strong coupling of a single photon to a superconducting qubit using circuit quantum electrodynamics, Nature 431, 162 (2004).

[63] M. Trupke, J. Goldwin, B. Darquié, G. Dutier, S. Eriksson, J. Ashmore and E. A. Hinds, Atom Detection and Photon Production in a Scalable, Open, Optical Microcavity, Phys. Rev. Lett. 99, 063601 (2007).

[64] Y. Colombe, T. Steinmetz, G. Dubois, F. Linke, D. Hunger and J. Reichel, Strong atomfield coupling for BoseEinstein condensates in an optical cavity on a chip, Nature 450, 272 (2007).

[65] R.J. Thompson, G. Rempe and H.J. Kimble, Observation of normal-mode splitting for an atom in an optical cavity, Phys. Rev. Lett. 68, 1132 (1992). 
[66] Q.A. Turchette, C.J. Hood, W. Lange, H. Mabuchi and H.J. Kimble, Measurement of Conditional Phase Shifts for Quantum Logic, Phys. Rev. Lett. 75, 4710 (1995).

[67] K.M. Birnbaum, A. Boca, R. Miller, A.D. Boozer, T.E. Northup and H.J. Kimble, Photon blockade in an optical cavity with one trapped atom, Nature 436, 87 (2005).

[68] N. Stefanou and M. Modinos, Impurity bands in photonic insulators, Phys. Rev. B 57, 12127 (1998).

[69] A. Yariv, Y. Xu, R.K. Lee and A. Scherer, Coupled-resonator optical waveguide: a proposal and analysis, Optics Lett. 24, 711 (1999).

[70] M. Marzani and D. Vanderbilt, Maximally localized generalized Wannier functions for composite energy bands, Phys. Rev. B 56, 12847 (1997).

[71] M.J. Hartmann, M. Reuter, and M.B. Plenio, Excitation and entanglement transfer versus spectral gap, New J. Phys. 8, 94 (2006).

[72] M.B. Plenio, J. Hartley and J. Eisert, Dynamics and manipulation of entanglement in coupled harmonic systems with many degrees of freedom, New J. Phys. 6, 36 (2004).

[73] M.P.A. Fisher, P.B. Weichman, G. Grinstein and D.S. Fisher, Boson localization and the superfluid-insulator transition, Phys. Rev. B 40, 546 (1989).

[74] M.P.A. Fisher and G. Grinstein and S. M. Girvin, Presence of quantum diffusion in two dimensions: Universal resistance at the superconductor-insulator transition, Phys. Rev. Lett. 64, 587 (1990).

[75] J. Hubbard, Electron Correlations in Narrow Energy Bands, Proc. Roy. Soc. A 276238 (1963).

[76] M. Fleischhauer, A. Imamoglu and J.P. Marangos, Electromagnetically induced transparency: Optics in coherent media, Rev. Mod. Phys. 77, 633 (2005).

[77] H. Schmidt and A. Imamoglu, Giant Kerr nonlinearities obtained by electromagnetically induced transparency, Opt. Lett. 21, 1936 (1996).

[78] A. Imamoglu, H. Schmidt, G. Woods and M. Deutsch, Strongly Interacting Photons in a Nonlinear Cavity, Phys. Rev. Lett. 79, 1467 (1997); P. Grangier, D.F. Walls and K.M. Gheri, Comment: Strongly Interacting Photons in a Nonlinear Cavity, Phys. Rev. Lett. 81, 2833 (1998); A. Imamoglu, H. Schmidt, G. Woods and M. Deutsch, Erratum: Strongly Interacting Photons in a Nonlinear Cavity, Phys. Rev. Lett. 81, 2836 (1998).

[79] M.J. Werner and A. Imamoglu, Photon-photon interactions in cavity electromagnetically induced transparency, Phys. Rev. A 61, 011801(R) (1999).

[80] K.M. Gehri, W. Alge and P. Grangier, Quantum analysis of the photonic blockade mechanism, Phys. Rev. A 60, R2673 (1999).

[81] S.M. Spillane, T.J. Kippenberg, K.J. Vahala, K.W. Goh, E. Wilcut and H.J. Kimble, Ultrahigh-Q toroidal microresonators for cavity quantum electrodynamics, Phys. Rev. A 71, 013817 (2005).

[82] F. Dimer, B. Estienne, A. S. Parkins and H. J. Carmichael, Proposed realisation of the Dicke-model quantum phase transition in an optical cavity QED system, Phys. Rev. A 75, 013804 (2007).

[83] A. Recati, P. O. Fedichev, W. Zwerger and P. Zoller, SpinCharge Separation in Ultracold Quantum Gases, Phys. Rev. Lett. 90, 020401 (2003).
[84] L.-M. Duan, E. Demler and M. D. Lukin, Controlling Spin Exchange Interactions of Ultracold Atoms in Optical Lattices, Phys. Rev. Lett. 91, 090402 (2003).

[85] M.A. Cazalilla and A.F. Ho, Instabilities in Binary Mixtures of One-Dimensional Quantum Degenerate Gases, Phys. Rev. Lett. 91, 150403 (2003).

[86] A. Imamoğlu, High Efficiency Photon Counting Using Stored Light, Phys. Rev. Lett. 89, 163602 (2002).

[87] D.F.V. James and P.G. Kwiat, Atomic-Vapor-Based High Efficiency Optical Detectors with Photon Number Resolution, Phys. Rev. Lett. 89, 183601 (2002).

[88] U. Schollwöck, The density-matrix renormalization group, Rev. Mod. Phys. 77, 259 (2005).

[89] J.K. Freericks and H. Monien, Strong-coupling expansions for the pure and disordered Bose-Hubbard model, Phys. Rev. B 53, 2691 (1996).

[90] T. Moriya and K. Ueda, Antiferromagnetic spin fluctuation and superconductivity, Rep. Prog. Phys. 66, 1299 (2003).

[91] H.J. Briegel and R. Raussendorf, Persistent Entanglement in Arrays of Interacting Particles, Phys. Rev. Lett. 86, 910 (2001).

[92] A.S. Sørensen and K. Mølmer, Entangling atoms in bad cavities, Phys. Rev. A 66, 022314 (2002).

[93] D.F.V. James, Quantum Computation with Hot and Cold Ions: An Assessment of Proposed Schemes, Fortschr. Phys. 48, 823 (2000).

[94] R. Raussendorf and H.J. Briegel, A One-Way Quantum Computer, Phys. Rev. Lett. 86, 5188 (2001).

[95] D. Gross and J. Eisert, Novel Schemes for MeasurementBased Quantum Computation, Phys. Rev. Lett. 98, 220503 (2006).

[96] K.M.R. Audenaert and M.B. Plenio, When are correlations quantum? - verification and quantification of entanglement by simple measurements, New J. Phys. 8, 266 (2006).

[97] S.G. Johnson and J.D. Joannopoulos, Photonic Crystals: The Road from Theory to Practice (Kluwer, 2002).

[98] D.G. Angelakis, P.L. Knight and E. Paspalakis, Photonic crystals and inhibition of spontaneous emission: an introduction, Cont. Phys. 45, 303 (2004).

[99] H. Altug and J. Vuckoic, Two-dimensional coupled photonic crystal resonator arrays, Appl. Phys. Lett. 84, 161 (2004).

[100] M. Bayindir, B. Temelkuran, and E. Ozbay, Propagation of photons by hopping: A waveguiding mechanism through localized coupled cavities in three-dimensional photonic crystals, Phys. Rev. B 61, R11855 (2000).

[101] M. Bayindir, B. Temelkuran and E. Ozbay, Tight-Binding Description of the Coupled Defect Modes in ThreeDimensional Photonic Crystals, Phys. Rev. Lett. 84, 2140 (2000).

[102] F.H. Julien and A. Alexandrou, Controlling Artificial Atoms, Science 20, 1429 (1998).

[103] A. Badolato, K. Hennessy, M. Atatüre, J. Dreiser, E. Hu, P.M. Petroff, Atac Imamoğlu, Deterministic Coupling of Single Quantum Dots to Single Nanocavity Modes, Sience 308, 1158 (2005).

[104] B.-S. Song, S. Noda, T. Asano and Y. Akahane, Ultra-highQ photonic double-heterostructure nanocavity, Nature Materials 4, 207 (2005).

[105] A.M. Armani, A. Srinivasan and K.J. Vahala, Soft Lithographic Fabrication of High Q Polymer Microcavity Arrays, Nano Letters 7, 6 (2007). 
[106] T.J. Kippenberg, S.M. Spillane, B. Min and K.J. Vahala, Demonstration of ultra-high-Q small mode volume toroid micro-cavities on a chip, Appl. Phys. Lett. 85, 6113 (2004).

[107] S.M. Spillane, T.J. Kippenberg, O.J. Painter and K.J. Vahala, Ideality in a Fiber-Taper-Coupled Microresonator System for Application to Cavity Quantum Electrodynamics, Phys. Rev. Lett. 91, 043902 (2003).

[108] B. Dayan, A.S. Parkins, T. Aoki, E.P. Ostby, K.J. Vahala and H.J. Kimble, A Photon Turnstile Dynamically Regulated by One Atom, Science 319, 1062 (2008).

[109] M. Trupke, E.A. Hinds, S. Eriksson, E.A. Curtis, Z. Moktadir, E. Kukharenka and M. Kraft, Microfabricated highfinesse optical cavity with open access and small volume, Appl. Phys. Lett. 87, 211106 (2005).

[110] http://www3.imperial.ac.uk/ccm/research/coldatoms/cavity

[111] M.A. Sillanpää, J.I. Park and R.W. Simmonds, Coherent quantum state storage and transfer between two phase qubits via a resonant cavity, Nature 449, 438 (2007).

[112] J. Majer, J.M. Chow, J.M. Gambetta, J. Koch, B.R. Johnson, J.A. Schreier, L. Frunzio, D.I. Schuster, A.A. Houck, A. Wallraff, A. Blais, M.H. Devoret, S.M. Girvin and R.J. Schoelkopf, Coupling superconducting qubits via a cavity bus Nature 449, 443 (2007).

[113] J.M. Fink, M. Göppl, M. Baur, R. Bianchetti, P.J. Leek, A. Blais and A. Wallraff, Climbing the JaynesCummings ladder and observing its $\sqrt{n}$ nonlinearity in a cavity QED system, Nature 454, 315 (2008). 